\title{
Aberrant Uncertainty Processing Is Linked to Psychotic-like Experiences, Autistic Traits and Reflected in Pupil Dilation During Probabilistic Learning
}

- Draft 30.05.2022. This paper has not been peer reviewed. -

\author{
Isabel V. Kreis
}

Department of Psychology, UiT - The Arctic University of Norway, Tromsø, Norway

Lei Zhang

Department of Psychology, UiT - The Arctic University of Norway

Social, Cognitive and Affective Neuroscience Unit, Department of Cognition, Emotion, and Methods in Psychology, Faculty of Psychology, University of Vienna, Vienna, Austria Matthias Mittner

Department of Psychology, UiT - The Arctic University of Norway, Tromsø, Norway Leonard Syla

Department of Psychology, UiT - The Arctic University of Norway, Tromsø, Norway Claus Lamm

Social, Cognitive and Affective Neuroscience Unit, Department of Cognition, Emotion, and Methods in Psychology, Faculty of Psychology, University of Vienna, Vienna, Austria Vienna Cognitive Science Hub, University of Vienna, Vienna, Austria Gerit Pfuhl

Department of Psychology, UiT - The Arctic University of Norway, Tromsø, Norway

\section{Author Note}

Isabel V. Kreis, Department of Psychology, UiT - The Arctic University of Norway; Lei Zhang, Department of Psychology, UiT - The Arctic University of Norway, and Social, Cognitive and Affective Neuroscience Unit, Department of Cognition, Emotion, and Methods in Psychology, Faculty of Psychology, University of Vienna; Matthias Mittner, Department of Psychology, UiT - The Arctic University of Norway; Leonard Syla, Department of Psychology, UiT - The Arctic University of Norway; Claus Lamm, Social, Cognitive and Affective 
Neuroscience Unit, Department of Cognition, Emotion, and Methods in Psychology, Faculty of Psychology, University of Vienna, and Vienna Cognitive Science Hub, University of Vienna; Gerit Pfuhl, Department of Psychology, UiT - The Arctic University of Norway.

Isabel V. Kreis is now at NORMENT (Norwegian Centre for Mental Disorders Research), Institute of Clinical Medicine, University of Oslo.

The study was conducted in accordance with the guidelines of the Declaration of Helsinki and approved by the internal ethics committee of the Department of Psychology at UiT - The Arctic University of Norway (reference number: 2017/1912). Some preliminary data was presented at the 2019 European Conference on Schizophrenia Research, Berlin, Germany. All anonymized data as well as the computational modelling code are available in an Open Science Framework repository (DOI 10.17605/OSF.IO/6XAB2; https://osf.io/6xab2/), as is a pre-print version of the manuscript (https://doi.org/10.31219/osf.io/nc2rx).

This research was supported by a grant from the Norwegian Research Council (FRIMEDBIO 262338) and the Vienna Science and Technology Fund (WWTF VRG13-007). We thank all participants for their time and effort, and Wibecke Nederhagen Hope for assistance during piloting and translation of the instructions.

Correspondence concerning this article should be addressed to Isabel V. Kreis, Oslo universitetssykehus HF, Seksjon for psykoseforskning, Ullevål sykehus - bygg 49, Postboks 4956, Nydalen, 0424 Oslo, Norway. E-mail: i.v.kreis@medisin.uio.no 


\begin{abstract}
Aberrant belief updating due to misestimation of uncertainty and an increased perception of the world as volatile (i.e. unstable) has been found in autism and psychotic disorders. Pupil dilation tracks events that warrant belief updating, likely reflecting the adjustment of neural gain. However, whether subclinical autistic or psychotic symptoms affect this adjustment and how they relate to learning in volatile environments remains to be unraveled. Here, we investigated the relationship between behavioral and pupillometric markers of subjective volatility (i.e. experience of the world as unstable), autistic traits, and psychotic-like experiences in 52 neurotypical adults with a probabilistic reversal learning task. Computational modelling revealed that participants with higher psychotic-like experience scores overestimated volatility in low-volatile task periods. This was not the case for participants scoring high on autistic-like traits, who instead showed a diminished adaptation of choice-switching behavior in response to risk. Pupillometric data indicated that individuals with higher autistic- or psychotic-like trait and experience scores differentiated less between events that warrant belief updating and those that do not when volatility was high. These findings are in line with misestimation of uncertainty accounts of psychosis and autism spectrum disorders and indicate that aberrancies are already present at the subclinical level.
\end{abstract}

Keywords: Volatility; Belief Updating; Psychosis; Pupillometry; Hidden Markov Model

\title{
General Scientific Summary
}

Making decisions and evaluating one's own beliefs involves assessing and dealing with uncertainty, e.g. regarding one's prior assumptions and new information. It has been suggested that this process is compromised in autism and schizophrenia spectrum disorders and could explain a range of their clinical core symptoms. This study supports this notion by 
demonstrating a link between abnormalities in how uncertainty is processed and autistic- and psychotic-like symptoms in healthy individuals. 


\section{Aberrant Uncertainty Processing Is Linked to Psychotic-like Experiences, Autistic Traits and Reflected in Pupil Dilation During Probabilistic Learning}

Making good decisions requires learning about which choices are most likely associated with a positive outcome and updating beliefs about these outcome probabilities if they change. This integral skill can be impaired in psychiatric disorders. Accordingly, maladaptive and increased choice-switching during reversal learning tasks (where outcome probabilities reverse over time) has been observed in both autism spectrum disorders (ASD; D'Cruz et al., 2013; Mussey et al., 2015; Solomon et al., 2011) and psychotic disorders like schizophrenia (SCZ; Culbreth et al., 2016; Kaplan et al., 2016; Li et al., 2014; Murray et al., 2008; Schlagenhauf et al., 2014; Waltz et al., 2013). This finding may be explained by an overestimation of environmental volatility (instability; Cole et al., 2020; Deserno et al., 2020; Lawson et al., 2017), which in reversal learning tasks is defined by the rate at which the outcome probabilities reverse. Evidently, an elevated belief about environmental volatility may increase a person's tendency to switch between two choices, as the probability for e.g. obtaining a reward for one choice over another might change over time. Consequentially, beliefs about outcome probabilities are updated at a higher rate and behavior becomes hyperflexible (Deserno et al., 2020). Some findings imply that individuals with ASD do not necessarily overestimate volatility but are more sensitive to it, with impaired performance under volatile as opposed to stable conditions (Goris et al., 2020; Robic et al., 2015). In addition, choice behavior is affected by the accuracy with which outcome probability (henceforth risk; Payzan-LeNestour \& Bossaerts, 2011) is learned and represented in the first place, which seems to be diminished in patients with SCZ (Murray et al., 2008; Waltz et al., 2013; Weickert et al., 2010) and individuals with ASD (Solomon et al., 2015). Taken together, this indicates aberrancies in the representation and processing of different types of uncertainty in both clinical groups. Interestingly, misestimation of uncertainty and skewed belief updating have been proposed to 
play a major role in the development of symptoms in both autism and psychotic disorders within the Bayesian brain framework (Fletcher \& Frith, 2009; Van de Cruys et al., 2014; van Schalkwyk et al., 2017). Specifically, symptoms may arise from false inferences about the world, which in turn are caused by alterations in ascribing uncertainty to prior beliefs and new sensory information (Adams et al., 2013; Powers et al., 2017). This may lead to delusions and hallucinations in psychosis (Adams et al., 2013; Fletcher \& Frith, 2009) and cause symptoms of sensory overload and oversensitivity to sensory stimulation in autism (Lawson et al., 2014).

Subjective representations of the different uncertainties that characterize a task environment (such as risk and volatility) can be captured with cognitive-computational models fitted to observed behavior, e.g. Bayesian inference models like the Hidden Markov Model (HMM), which has successfully been applied to reversal learning tasks (Hämmerer et al., 2019; Schlagenhauf et al., 2014). In the HMM, subjective volatility is reflected by the transition probability, which describes the probability to switch between different belief states about risk within the task. Based on those state beliefs, two task-relevant trial-wise latent variables can be derived: choice uncertainty and Bayesian surprise. Choice uncertainty is high under high risk (i.e. when outcome probabilities are uniform) and when estimation uncertainty of the current risk is high (e.g. at the beginning of a new learning sequence or under high volatility). Bayesian surprise expresses the extent to which a current state belief should be updated and is particularly high under high volatility (Hämmerer et al., 2019). Both uncertainty and surprise can prompt belief updating, indicating a general need to 'learn more' and 'how much more', respectively.

Neurobiologically, uncertainty is thought to be encoded by neuromodulatory systems, where contextual change resulting from volatility may specifically be signaled by norepinephrine (Friston et al., 2006; Yu \& Dayan, 2005). This fits well with accounts linking norepinephrinergic activity in the locus coeruleus (the LC-NE system) to explorative behavior (Aston-Jones \& Cohen, 2005), given that contextual change promotes belief updating through 
exploration and learning. Activity in the LC-NE system can be indexed through pupil size (Joshi et al., 2016; Rajkowski et al., 1994; Samuels \& Szabadi, 2008), which in turn has been found to respond to (choice) uncertainty (Kreis et al., 2021; Nassar et al., 2012), environmental volatility (Browning et al., 2015; Lawson et al., 2017), surprise and belief updating (Hämmerer et al., 2019; Preuschoff et al., 2011). This response possibly reflects an upregulation of neural gain and learning (Eldar et al., 2013) to reduce uncertainty about current task states and update beliefs accordingly. Pupil responses in individuals with autism (Lawson et al., 2017) or schizophrenia (Kreis et al., 2021; Steinhauer \& Zubin, 1982; Steinhauer et al., 1979), however, seem to scale less with uncertainty or surprise, suggesting a reduced ability to differentiate events that warrant a belief update from those that do not and to regulate neural gain accordingly. This may be caused by a failure to represent task structures appropriately (Hämmerer et al., 2019), e.g. by misestimating risk, enhanced estimation uncertainty due to diminished learning, or overestimation of volatility which renders all new events similarly surprising and relevant for belief updating.

It is unclear to what extent these findings translate to neurotypical populations varying naturally on autistic- and psychotic-like symptoms (Abu-Akel et al., 2015; Yung et al., 2009) and whether the effects scale with symptom load. The study of sub-clinical populations is essential when evaluating the potential role of uncertainty misestimation for symptom development as described above. Furthermore, findings in patient samples may be tainted by the effects of e.g. anticholinergic medication on pupil size (Naicker et al., 2016). Hence, the present study set out to test whether autistic- and psychotic-like traits and experiences assessed in a neurotypical sample are associated with similarly reduced pupil responses to events that should promote belief updating. Such an association may indicate an increased exploratory processing style, possibly resulting from elevated subjective environmental volatility. Using a probabilistic prediction task with different volatility and risk conditions, latent computational 
variables (subjective volatility, choice uncertainty, Bayesian surprise) were derived from computational models and tested for their relationship with trait and experience scores and with changes in pupil size. Trait and experience scores as well as task conditions were further investigated in their relation to observable behavior such as accuracy of and switching between predictions, both of which were expected to differ depending on the degrees of environmental volatility and risk. Interaction effects between these task-related uncertainty conditions and trait scores would then help to clarify whether different traits are related to issues in dealing with volatility (e.g. reflected in particularly decreased performance in the volatile condition), misestimation of volatility (e.g. reflected in similar amounts of switching under more and less volatile conditions) or misestimation of risk (e.g. reflected in similar amounts of switching under high and low risk conditions). Working memory capacity was evaluated to ensure that trait-related differences in probabilistic learning were not driven by differences in executive functioning and working memory resources, which have been linked to learning about probabilities and task structures (Deserno et al., 2020; Waltz \& Gold, 2007).

\section{Method}

\section{Participants}

Participants were recruited through pamphlets, social media, and from university classes. Inclusion criteria were: (1) 18 - 60 years of age, (2) normal or corrected-to-normal eyesight, (3) no history of neurological disorders, (4) no acute psychiatric disorder, (5) no substance dependence, and (6) no intake of any psychoactive medication or recreational drug within three months prior to the assessment. The final sample consisted of 52 individuals and is described in Table 1 . The sample size was based on a power analysis ( $\alpha=0.05$, two-sided, $1-\beta=0.8$ ) leaned on recent findings of an association between pupil response to uncertainty and performance in a volatile task environment $(\mathrm{r}=0.62, \mathrm{~N}=22$; de Berker et al., 2016). Effect 
size and final sample size were slightly reduced (to 0.4 ) and increased (to $\mathrm{N}=52$ ) respectively to account for publication bias and take potential participant exclusion due to eye-tracking data quality into account. For all participants, written informed consent was obtained prior to the assessment. The study was conducted in accordance with the guidelines of the Declaration of Helsinki and approved by the internal ethics committee of the Department of Psychology at UiT - The Arctic University of Norway (reference number: 2017/1912).

\section{Table 1}

Summary Statistics of Demographic Variables, Average Questionnaire Scores and Working Memory Capacity

\begin{tabular}{llll} 
& $n$ & $M(S D)$ & $M d(I Q R)$ \\
\hline Gender (f/m) & $31 / 21$ & & \\
Education (HS/BA/MA) & $34 / 13 / 5$ & & \\
Age & & $23.50(4.13)$ & $22.50(6.00)$ \\
CAPE-P & & $1.46(0.30)$ & $1.41(0.50)$ \\
AQ & & $0.30(0.15)$ & $0.29(0.21)$ \\
WMC & & \\
& verbal-numerical & $3.92(0.90)$ & $4.00(1.00)$ \\
& visual-spatial & $6.13(0.84)$ & $6.00(1.25)$ \\
\hline
\end{tabular}

Notes. $n=$ sample size of the different levels of categorical variables; $M=$ mean; $S D=$ standard deviation; $M d=$ median; $I Q R=$ interquartile range; $\mathrm{f}=$ female, $\mathrm{m}=$ male; $\mathrm{HS}=$ high school, $\mathrm{BA}=$ bachelor, $\mathrm{MA}=$ master; CAPE-P $=$ average score of the positive dimension scale of the CAPE (Community Assessment of Psychic Experiences), maximum score $=4$, minimum $=1 ; \mathrm{AQ}=$ average score of the Autism Quotient, maximum score $=1$, minimum $=0 ; \mathrm{WMC}=$ working memory capacity score, maximum score $=7$, minimum $=0$. Results are rounded to two decimal places. 


\section{Materials and Procedure}

Probabilistic prediction task. A probabilistic prediction task programmed in Psychopy (Peirce et al., 2019) was administered to measure decision-making and belief updating under different uncertainty conditions. Two task blocks ('volatile' and 'cued') differed in their degree of volatility, compromising 160 trials each $(+12$ and 18 training trials for the volatile and the cued task block, respectively). On each trial a vertically striped stimulus was presented in the center of the screen, followed by an either left- or right-tilted stimulus (orientation $\pm 45^{\circ}$ ). Upon presentation of the vertical stimulus, participants had to indicate via key -press (left-alt: 'lefttilted', right-ctrl: 'right-tilted') which one of the two tilted stimuli they predicted to see next (Fig. 1a). After a two-second delay, the outcome (left- or right-tilted stimulus) was displayed for two seconds. The probability of seeing either a left- or a right-tilted stimulus was unknown to the participants and changed every $20( \pm 4)$ trials, alternating between 80:20 and 60:40 and their reverse $(20: 80,40: 60)$, providing task periods of high $(60: 40,40: 60)$ and low $(80: 20$, 20:80) risk and inducing high estimation uncertainty after each change (Fig. 1b). Changes in the underlying distribution of left- and right-tilted stimuli were not announced in the volatile, and announced in the cued block (see Fig. 1a). The order of the different risk conditions was the same across blocks and participants, while the identity of the majority stimulus was inverted between blocks (Fig. 1c). Block order was not counterbalanced (i.e., the volatile task block came always first) to prevent priming participants from detecting the hidden change points in the volatile task block. Participants were instructed to fixate the center of the screen throughout the task and minimize the total amount of prediction errors. They were informed that over several trials in a row either the left- or the right-tilted stimulus would appear more often with a fixed but unknown probability, that probability and identity of the majority stimulus might change repeatedly, and that these changes would be hidden in the first, but announced in the second task block. Participants were advised to forget all they had learned about the stimulus 
probabilities on previous trials and start to learn 'anew' following a change announcement. Task performance was assessed as accuracy (relative frequency of predicting the current majority stimulus) and proportion of choice switches (proportion of times where prediction on trial $t+1$ was different from prediction on trial $t$; Fig. 1c), both aggregated separately for the two risk conditions per block.

\section{Figure 1}

The Probabilistic Prediction Task

a

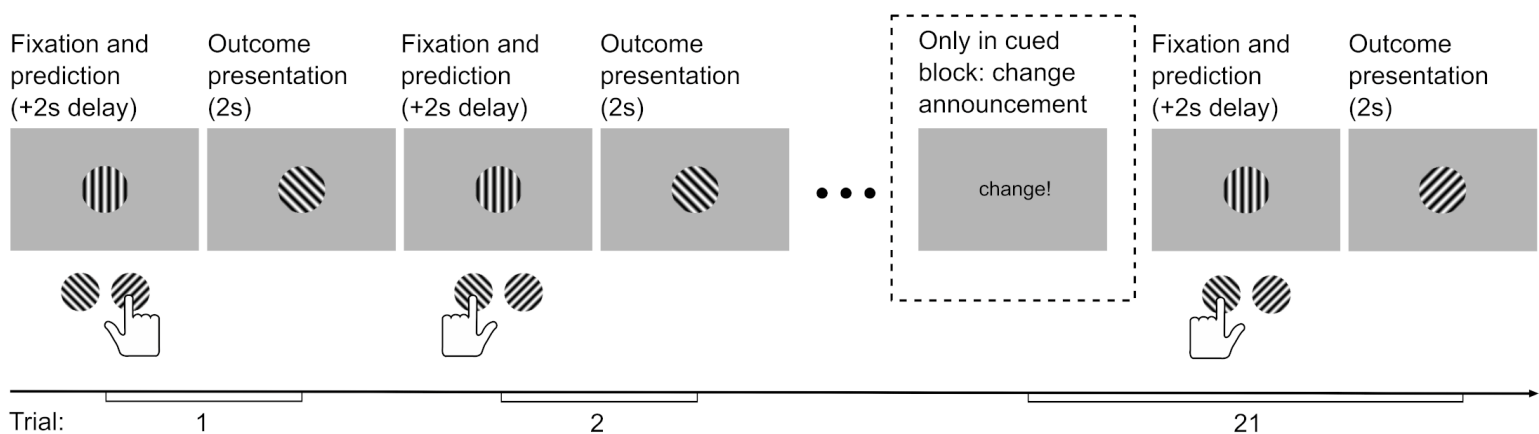

b

C
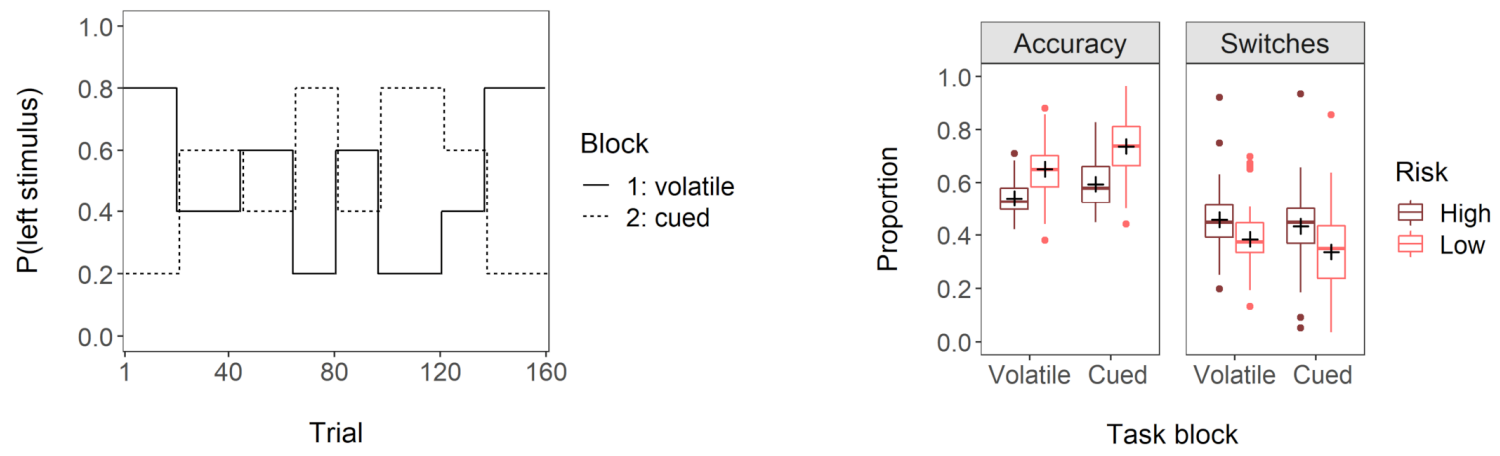

Notes. (A) Example trials with a change of stimulus probabilities on trial 21. In the second, cued task block, this change was preceded by a 'change' message on screen. In response, participants had to press 'enter' before they could continue with the task. (B) Task structure: probabilities for the left- $\left(\mathrm{p}_{\text {left }}\right)$ and the right-tilted (1-p $\left.\mathrm{p}_{\text {left }}\right)$ stimulus in the first task block (volatile block; solid line) and the second task block (cued block; dashed line). Time points of changes were identical in both blocks (lines are slightly jittered for better readability), as was the order of the different risk conditions. The identity of the majority stimulus in the different $<$ risk conditions was inverted in the second as opposed to the first task 
block. (C) Boxplots displaying the proportion of trials where the majority stimulus was predicted (accuracy) and where choices differed from those on the preceding trial (switches) for the different task blocks and risk conditions, respectively. Means are displayed as crosses.

Questionnaires and working memory measure. Autistic-like traits and psychotic-like experiences were measured with the abridged version of the Autism Quotient (AQ; Hoekstra et al., 2011), and the positive symptom dimension of the Community Assessment of Psychic Experiences (CAPE-P; Stefanis et al., 2002), respectively. For four participants, responses to three items of the CAPE-P (15\% of all items) were missing, thus CAPE-P and AQ average scores are used in the main analyses. Additionally, age, gender, and education (recorded in categories of highest completed degree: high school, Bachelor, Master) were recorded. Working memory capacity was assessed in the verbal-numerical domain and the visual-spatial domain, using the digit span and the matrix span task of a computerized open source working memory test battery (Stone \& Towse, 2015; see Supplementary Methods for details).

Pupil size. During the prediction task, pupil diameter was recorded from the right eye at a sampling rate of $500 \mathrm{~Hz}$ with an infrared video-based eye tracker (Eyelink 1000, SR Research).

\section{Procedure}

On the day of the assessment, participants signed the consent form before completing the first block of the prediction task (ca. $15 \mathrm{~min}$ ). Then, the working memory task (ca. $10 \mathrm{~min}$ ), a decision-making task (ca. 5 min; results are not reported here and are not assumed to affect behavior on any of the other tasks), and the second block of the prediction task (ca. 15 min) were administered. Finally, participants filled out the questionnaires (ca. $10 \mathrm{~min}$ ). 


\section{Analysis}

Computational modelling of behavior. To quantify latent cognitive processes, seven candidate computational models were fitted independently to participants' choices for the volatile and the cued block of the prediction task, respectively. The models included a simple win-stay-lose-shift model (Worthy \& Todd Maddox, 2014), four different Reinforcement Learning models (den Ouden et al., 2013; Gläscher et al., 2008; Pearce \& Hall, 1980; Rescorla \& Wagner, 1972) and two variants of the Hidden Markov Model (HMM; Schlagenhauf et al., 2014). All models were estimated under the hierarchical Bayesian framework (Ahn et al., 2017; Gelman et al., 2013; Zhang et al., 2020) using a Hamiltonian Monte Carlo (HMC) method within the statistical language Stan. See Supplementary Methods for details, including model comparison and rationale behind the choice of models. Model comparison revealed that a variant of the HMM provided the best fit for both task blocks (see Supplementary Table S1 and S2). The HMM is a Bayesian inference model which assumes that participants make their choices (i.e. predict 'left' or 'right') based on their belief of being in a state of the task where either the left- or the right-tilted stimulus is more common. Those beliefs are updated on a trialby-trial basis, modulated by the history of action-outcome pairs and the estimated transition probability for the two states (i.e. how likely does the state change from 'predominantly left' to 'predominantly right-tilted stimuli' and vice versa). Crucially, the transition probability $\gamma$ reflects the perceived, i.e. subjective, volatility of the environment. In the winning variant of the HMM, effects of positive (prediction correct) vs. negative feedback (prediction incorrect) on state belief updates were allowed to differ (HMMRP; Schlagenhauf et al., 2014). For the cued task block, this model included belief resets at every announced change point. 
Based on the HMMRP's trial-wise state beliefs both before $\left(P\left(S_{t_{p r e}}\right)\right)$ and after observing the outcome $\left(P\left(S_{t_{\text {post }}}\right)\right)$, a Bayesian surprise signal was estimated as their KullblackLeibler (KL) divergence:

$$
D_{K L}\left(P\left(S_{t_{\text {post }}}\right) \| P\left(S_{t_{\text {pre }}}\right)\right)=\sum_{i=1}^{2} P\left(S_{t_{\text {pre }}}=i\right) \log \left(\frac{P\left(S_{t_{\text {post }}}=i\right)}{P\left(S_{t_{\text {pre }}}=i\right)}\right) \quad \text { Eq. } 1
$$

This expresses the extent to which the internal model (i.e. belief about the state) should be updated on each trial.

Similarly, choice uncertainty regarding the chosen stimulus was derived for different posterior 'reward' (i.e. a correct prediction) probabilities as belief entropy:

$$
H\left(S_{t}\right)=-\sum_{i=1}^{2} P\left(S_{t}=i\right) \log P\left(S_{t}=i\right)
$$

Pupil signal preprocessing. Eye blinks and other artifacts (e.g. caused by head movements or eye lid flickering) were detected with a custom-built filter based on the pupil signal's velocity implemented in R (version 3.5.1; R Core Team, 2018), and removed through cubic-spline interpolation (Mathôt et al., 2018). Velocity thresholds and margins for blink windows were adapted on an individual basis to account for inter-individual differences in blink characteristics, e.g. regarding the speed of signal recovery. The corrected pupil signal was smoothed with a low pass Butterworth filter using a cut-off frequency of $3 \mathrm{~Hz}$, since high frequency components are more likely caused by noise (Klingner et al., 2008). When the time window of interpolation spanned more than 1,000 consecutive milliseconds, the signal was treated as missing. The smoothed pupil signal was z-scored per block and participant and baseline-corrected per trial by subtracting the average signal of the $500 \mathrm{~ms}$ preceding the outcome onset. Trials with more than $50 \%$ of interpolated and missing data within the baseline or outcome presentation time window were treated as missing in subsequent analyses, where maximum pupil dilation during outcome presentation was the main variable of interest. 
Statistical analyses. Linear mixed-effects models were used to investigate the effect of task conditions (high risk: 60:40/40:60 trials vs. low risk: 80:20/20:80 trials; cued block vs. volatile block), AQ and CAPE-P scores on accuracy, choice switches, entropy and Bayesian surprise. In all models, nested random factors were specified, allowing for different intercepts at the different levels of risk condition within blocks nested within participants. The effect of entropy, Bayesian surprise, AQ and CAPE-P scores on pupil dilation were also tested using linear mixed-effects models. Model residuals were tested for normality and dependent variables were cube-root (Bayesian surprise) or square-root transformed (maximum pupil dilation) when this assumption was violated. Since autistic traits and psychotic-like experiences are positively correlated (Bevan Jones et al., 2012; Martinez et al., 2020), analyses were implemented separately for AQ and CAPE-P. Non-normally distributed variables were identified with Shapiro-Wilk tests and to evaluate the relationship between questionnaire scores and potential covariates, Spearman correlations (age; verbal-numerical and visual-spatial working memory scores), Kruskal-Wallis (education) and Mann-Whitney U tests (gender) were carried out. All testing was conducted two-sided and with a significance level of 0.05 .

\section{Transparency and openness}

This study was not pre-registered. All measures, data exclusions, and determination of the sample size are reported in the preceding sub-sections. All anonymized data as well as the computational modelling code are available in an Open Science Framework repository: DOI 10.17605/OSF.IO/6XAB2 (https://osf.io/6xab2/). Data was analyzed using the statistical programming language R (version 3.5.1; R Core Team, 2018), with R packages $n$ lme (version 3.1-152; Pinheiro et al., 2021) for linear-mixed effects models and ggplot2 for visualization 
(version 3.3.5, Wickham, 2016). Standardized regression coefficients are reported together with $95 \%$ confidence intervals. All results are rounded to two decimal places.

\section{Results}

AQ and CAPE-P scores were positively but not significantly correlated $(\rho=0.25, p=$ .08). Neither demographic, nor working memory variables were related to questionnaire scores (see Supplementary Results) and therefore not included as covariates in any of the subsequent analyses.

\section{Accuracy differs by task conditions but not questionnaire scores}

Accuracy was higher in the cued task block $(\beta=0.69, t=5.40, p<.001,95 \%$ CI $[0.43$, 0.94]) and lower in the high-risk condition $(\beta=-0.90, t=-8.57, p<.001,[-1.10,-0.69]$; Fig. 1c), with no significant interaction between block and risk $(\beta=-0.25, t=-1.69, p=.09,[-0.54$, 0.04]). When included in the model, none of the AQ predictors yielded a significant effect (AQ: $\beta=-0.10, t=-0.92, p=.36,[-0.33,0.12]$; block*AQ: $\beta=0.04, t=0.29, p=.77,[-0.22,0.29]$; risk*AQ: $\beta=0.08, t=0.78, p=.44,[-0.12,0.29]$; block*risk*AQ: $\beta=-0.16, t=-1.06, p=.29$, [-0.45, 0.13]; Fig. 2). Results of a model including CAPE-P instead of AQ scores were similar (CAPE-P: $\beta=-0.12, t=-1.06, p=.29,[-0.34,0.10]$; block ${ }^{*}$ CAPE-P: $\beta=0.00, t=-0.01, p=$ $.99,[-0.25,0.25]$; risk*CAPE-P: $\beta=0.17, t=1.68, p=.10,[-0.03,0.38]$; block*risk*CAPE-P $(\beta=-0.23, t=-1.59, p=.12,[-0.52,0.05]$; Fig. 2) 


\section{Figure 2}

Relationship Between Trait and Experience Scores and Proportion of Accurate Predictions
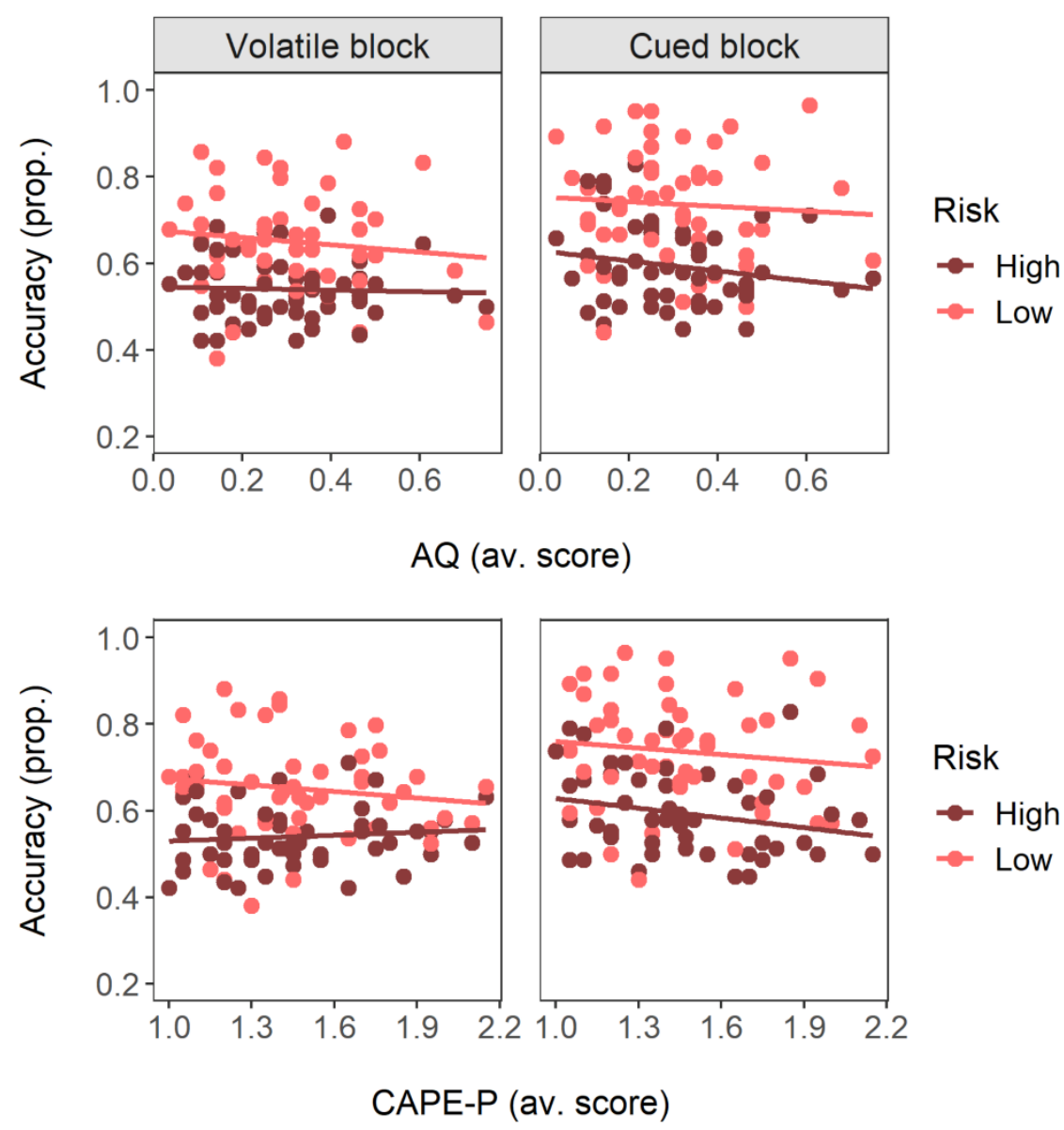

Notes. Proportion of accurate predictions is presented separately for the different task blocks (columns) and risk conditions (color). Trait and experience scores are average scores of AQ (top row) and CAPEP (bottom row).

\section{Choice switches differ by task conditions and AQ scores}

Proportion of choice switches was lower in the cued task block $(\beta=-0.33, t=-2.76, p$ $<.01,95 \%$ CI $[-0.56,-0.09])$ and higher for high-risk trials $(\beta=0.50, t=5.80, p<.001,[0.33$, 0.67]; Fig. 1c), with no significant interaction between block and risk $(\beta=0.16, t=1.27, p=$ $.21,[-0.08,0.40])$. Inclusion of AQ scores revealed a significant interaction with risk $(\beta=-$ $0.19, t=-2.22, p=.03,[-0.36,-0.02])$, indicating that proportion of switches on high- vs. low- 
risk trials differed less for participants scoring higher on the AQ (Fig. 3). Other AQ predictors were not significant (AQ: $\beta=0.18, t=1.39, p=.17,[-0.08,0.45]$; block*AQ: $\beta=-0.06, t=-$ $0.47, p=.64,[-0.29,0.18]$; block*risk*AQ: $\beta=0.11, t=0.87, p=.39,[-0.13,0.34])$. None of the CAPE-P predictors were significant (CAPE-P: $\beta=0.09, t=0.69, p=.49,[-0.17,0.35]$; block*CAPE-P: $\beta=0.09, t=0.73, p=.47,[-0.15,0.32]$; risk*CAPE-P: $\beta=0.03, t=0.30, p=$ $.77,[-0.14,0.20]$; block*risk*CAPE-P: $\beta=-0.02, t=-0.13, p=.90,[-0.26,0.23]$; Fig. 3$)$.

\section{Figure 3}

Relationship Between Trait and Experience Scores and Proportion of Choice Switches
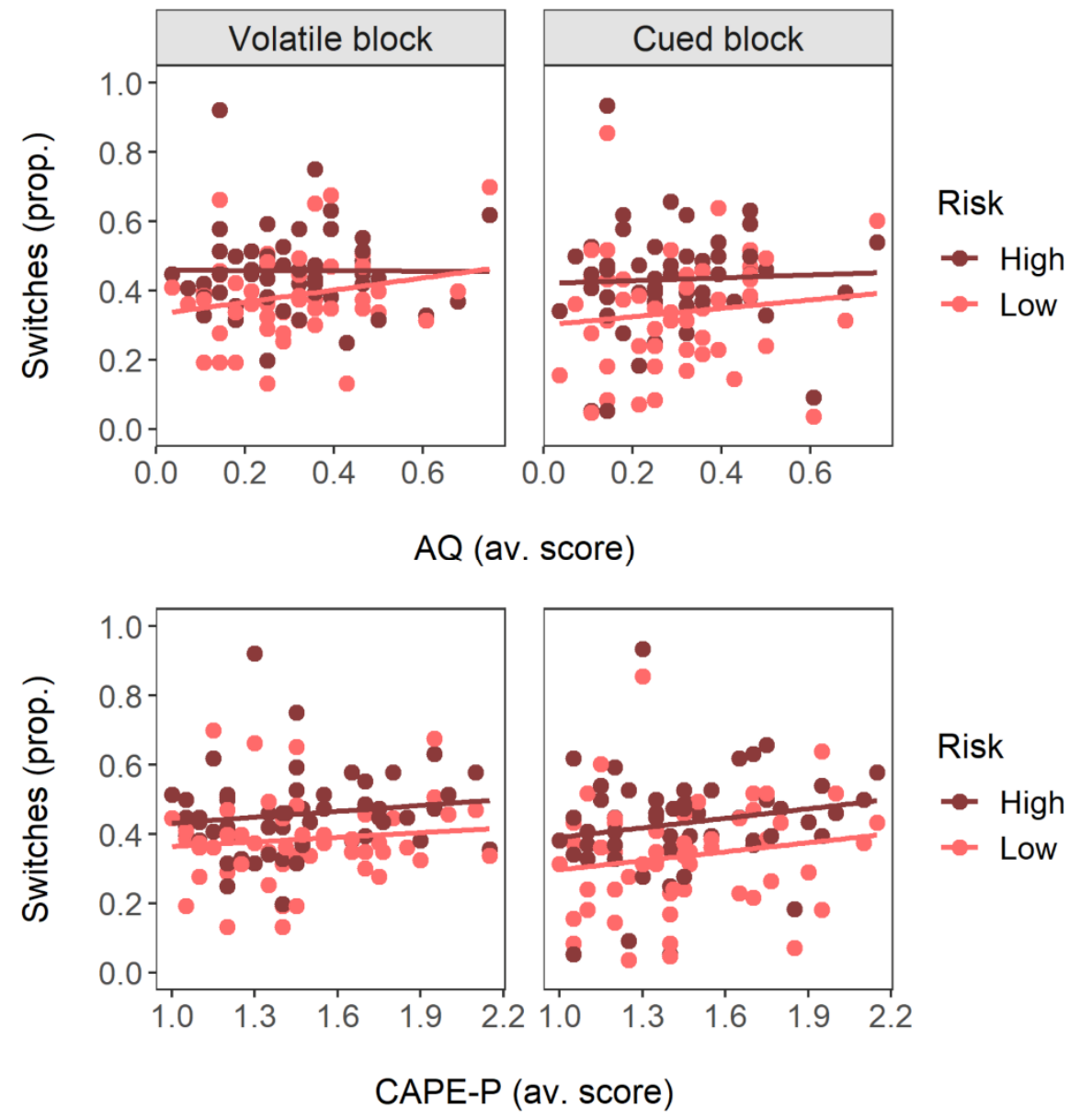

Notes. Proportion of choice switches is presented separately for the different task blocks (columns) and risk conditions (color). Trait and experience scores are average scores of AQ (top row) and CAPE-P (bottom row). 


\section{Transition probability $\left(\mathrm{HMM}_{\mathrm{RP}}\right)$ differs by block and CAPE-P scores}

Since $\mathrm{HMM}_{\mathrm{RP}}$ models were fitted separately per task block and differed slightly in terms of belief resets, the estimated transition probabilities were contrasted by directly comparing their posterior distributions. Transition probability $\gamma$ (subjective volatility) was credibly higher in the volatile block $(M=0.22)$ than in the cued block $(M=0.10)$ of the task $(95 \%$ highest density interval of the difference $[0.05,0.19])$. AQ scores were not significantly related to $\gamma$ in either block (volatile block: $\rho=0.17, p=.23$; cued block: $\rho=0.25, p=.08$ ), whereas CAPE-P scores and $\gamma$ correlated positively within the cued block $(\rho=0.28, p=.04$; volatile block: $\rho=$ $0.16, p=.25)$, indicating that participants with more psychotic-like experiences assumed higher volatility in the low-volatile block.

\section{Pupil response to entropy and Bayesian surprise is modulated by AQ and CAPE-P scores}

Both entropy (choice uncertainty) and Bayesian surprise differed by task conditions but were unrelated to questionnaire scores. Both measures had higher values under high risk, Bayesian surprise was increased under high volatility, and the effect of risk on entropy was more pronounced under low volatility (see Supplementary Results). The pupil response to trialby-trial entropy and Bayesian surprise (z-scored per participant and block) was assessed in two separate models. Here, maximum pupil dilation during outcome presentation (square root transformed) was significantly larger on trials where entropy was higher $(\beta=0.02, t=2.16, p$ $=.03,95 \%$ CI $[0.00,0.05]$ ), independent of block (block: $\beta=0.06, t=1.26, p=.22,[-0.04$, 0.17]; entropy*block: $\beta=0.01, t=0.41, p=.68,[-0.03,0.04])$, and similarly, increased with Bayesian surprise $(\beta=0.04, t=3.22, p<.01,[0.01,0.06])$, with no block effects (block: $\beta=$ $0.06, t=1.25, p=.22,[-0.04,0.17]$; Bayesian surprise*block: $\beta=-0.03, t=-1.55, p=.12,[-$ $0.06,0.01]$ ). However, these effects changed when adding questionnaire scores to the models, 
with responses to Bayesian surprise but not entropy affected by AQ (Table 2) and responses to entropy but not Bayesian surprise affected by CAPE-P (Table 3).

\section{Table 2}

Linear Mixed-Effects Model Results for Pupil Dilation (Dependent Variable) by Entropy

(Choice Uncertainty), Bayesian Surprise and $A Q$

\begin{tabular}{|c|c|c|c|c|c|c|c|}
\hline $\begin{array}{l}\text { Latent } \mathrm{HMM}_{\mathrm{RP}} \\
\text { predictor }\end{array}$ & & $\beta$ & $t$ & $p$ & $\mathrm{Cl}(95 \%)$ & $R^{2}{ }_{M}$ & $R^{2}{ }_{C}$ \\
\hline \multirow[t]{8}{*}{ Entropy } & & & & & & 0.01 & 0.16 \\
\hline & Entropy & 0.02 & 2.16 & .03 & {$[0.00,0.05]$} & & \\
\hline & block & 0.06 & 1.30 & .20 & {$[-0.03,0.16]$} & & \\
\hline & $\mathrm{AQ}$ & -0.01 & -0.14 & .89 & {$[-0.12,0.11]$} & & \\
\hline & Entropy*block & 0.01 & 0.41 & .68 & {$[-0.03,0.04]$} & & \\
\hline & Entropy*AQ & 0.00 & -0.06 & .95 & {$[-0.02,0.02]$} & & \\
\hline & Block*AQ & 0.10 & 1.99 & .05 & {$[0.00,0.19]$} & & \\
\hline & Entropy*block*AQ & 0.01 & 0.47 & .64 & {$[-0.02,0.04]$} & & \\
\hline \multirow{8}{*}{$\begin{array}{l}\text { Bayesian } \\
\text { surprise }\end{array}$} & & & & & & 0.01 & 0.16 \\
\hline & Bay. surprise & 0.04 & 3.24 & $<.01$ & {$[0.01,0.06]$} & & \\
\hline & block & 0.06 & 1.30 & .20 & {$[-0.03,0.16]$} & & \\
\hline & $\mathrm{AQ}$ & -0.01 & -0.14 & .89 & {$[-0.12,0.11]$} & & \\
\hline & Bay. surprise*block & -0.03 & -1.57 & 0.12 & {$[-0.06,0.01]$} & & \\
\hline & Bay. surprise*AQ & -0.02 & -2.17 & .03 & {$[-0.05,0.00]$} & & \\
\hline & Block*AQ & 0.10 & 1.98 & .05 & {$[0.00,0.19]$} & & \\
\hline & $\begin{array}{l}\text { Bay. surprise*block* } \\
\text { AQ }\end{array}$ & 0.05 & 3.07 & $<.01$ & {$[0.02,0.08]$} & & \\
\hline
\end{tabular}

Notes. Coefficients of the fixed effects in the separate linear mixed-effects models of pupil dilation by entropy and Bayesian surprise (both z-scored per block and participant; model included nested random effects for block within participant), including AQ score as a predictor; block = contrast of the second, cued task block to the first, volatile task block; $\mathrm{AQ}=$ Autism Quotient average score; $R^{2} m=$ marginal $R^{2}$, i.e. proportion of variance explained by the fixed effects alone; $R^{2} c=$ conditional $R^{2}$, i.e. proportion of variance explained by both the fixed and random effects $\left(R^{2} m\right.$ and $R^{2} c$ based on Nakagawa \& Schielzeth, 2013). Results are rounded to two decimal places. 
Specifically, inclusion of AQ scores revealed a significant three-way interaction with Bayesian surprise and block $(\beta=0.05, t=3.07, p<.01,[0.02,0.08])$, indicating reduced differentiation between high and low surprise values in the volatile, and increased differentiation in the cued task block for individuals with higher AQ scores (Fig. 4, Supplementary Fig. S2).

\section{Figure 4}

Pupil Responses to Entropy and Bayesian Surprise, Moderated by AQ Scores
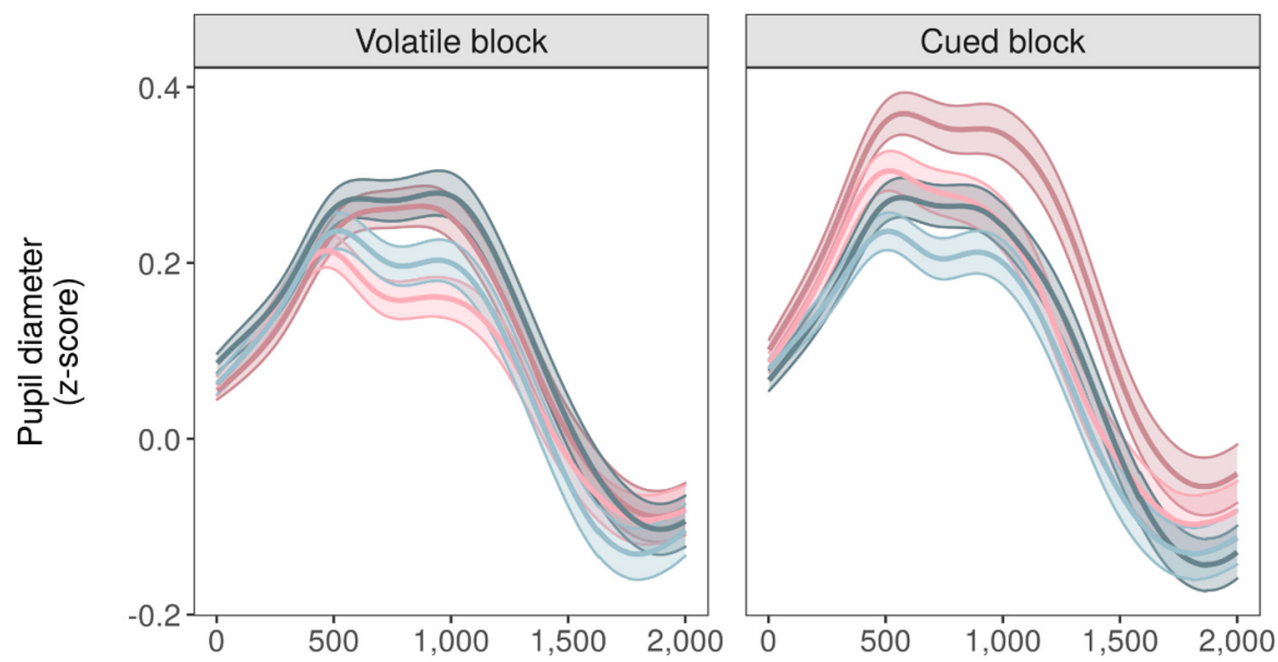

$A Q$ score \& Entropy
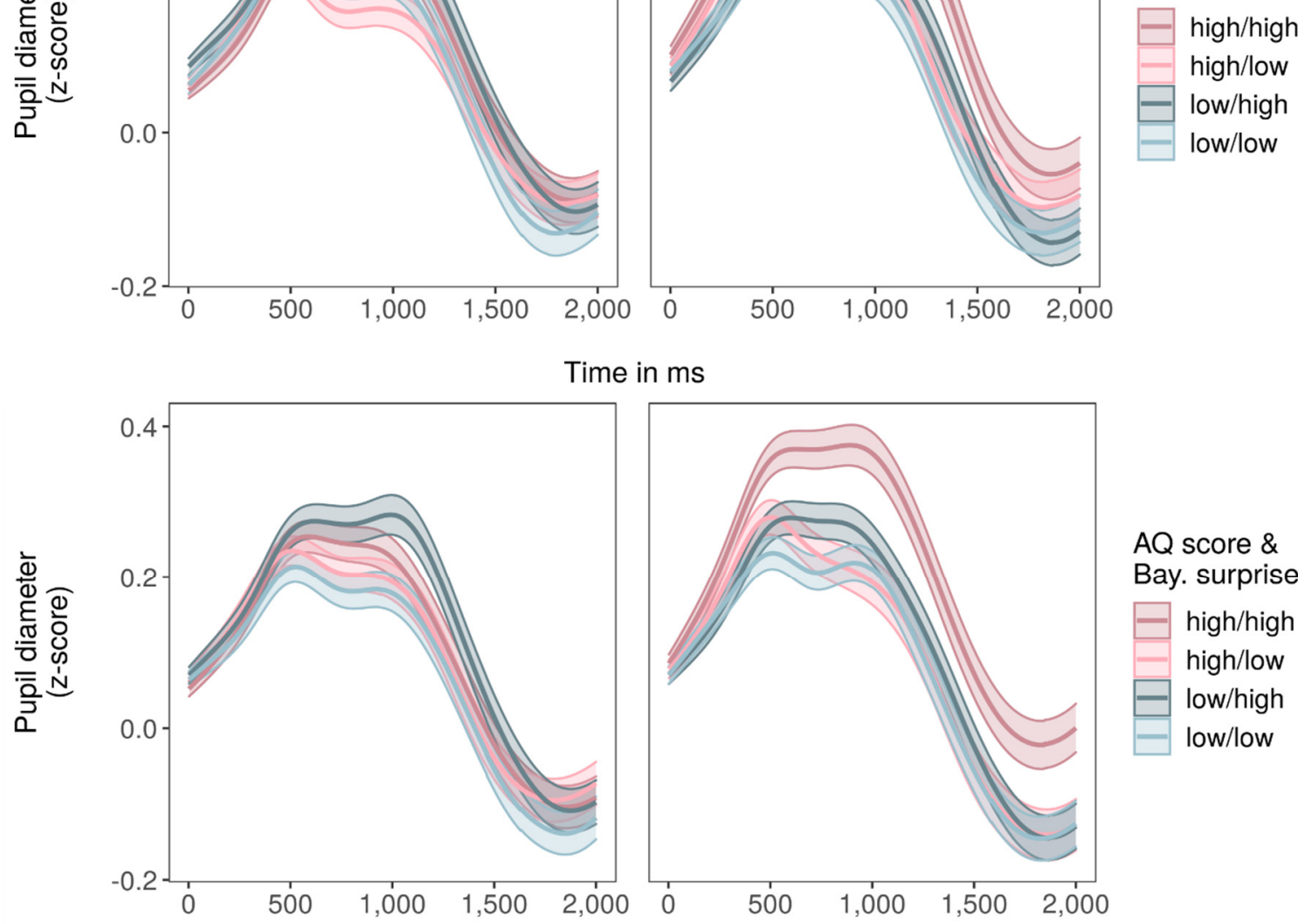

$A Q$ score \& Bay. surprise high/high high/low low/high low/low

Time in ms

Notes. Pupil responses during outcome presentation to entropy (top row) and Bayesian surprise (bottom row) are presented separately for the two task blocks (columns). Colors differentiate between responses 
for trials with high or low entropy (defined as values within participant-specific upper and lower quartile) and participants scoring high or low on the AQ (defined as values above or below the samplebased median). Red-ish colors indicate a high, blue-ish colors a low AQ score, darker shades represent high, brighter shades low entropy/Bayesian surprise values. Mean (solid line) and standard error of the mean (shaded area) were calculated for each sample of the z-scored and baseline-corrected pupil signal during outcome presentation. The quartile- and median-based categorizations were not used in any of the statistical models and only applied here to increase readability of the figure. 
In contrast, inclusion of CAPE-P scores (Table 3) yielded a significant three-way interaction with entropy and block $(\beta=0.04, t=2.22, p=.03,[0.01,0.07])$, indicating a diminished pupil response to entropy in individuals with higher CAPE-P scores within the volatile task block (Fig. 5, Supplementary Fig. S3).

\section{Table 3}

Linear Mixed-Effects Model Results for Pupil Dilation by Entropy (Choice Uncertainty), Bayesian Surprise and CAPE-P

\begin{tabular}{|c|c|c|c|c|c|c|c|}
\hline $\begin{array}{l}\text { Latent } \mathrm{HMM}_{\mathrm{RP}} \\
\text { predictor }\end{array}$ & & $\beta$ & $t$ & $p$ & $\mathrm{Cl}(95 \%)$ & $R^{2}{ }_{M}$ & $R^{2}{ }_{C}$ \\
\hline \multirow[t]{8}{*}{ Entropy } & & & & & & 0.01 & 0.16 \\
\hline & Entropy & 0.02 & 2.05 & .04 & {$[0.00,0.05]$} & & \\
\hline & block & 0.06 & 1.26 & .21 & {$[-0.04,0.17]$} & & \\
\hline & C-P & -0.11 & -2.07 & .04 & {$[-0.23,0.00]$} & & \\
\hline & Entropy*block & 0.01 & 0.50 & .62 & {$[-0.02,0.04]$} & & \\
\hline & Entropy*C-P & -0.02 & -1.77 & .08 & {$[-0.04,0.00]$} & & \\
\hline & Block*C-P & 0.01 & 0.21 & .83 & {$[-0.09,0.11]$} & & \\
\hline & Entropy*block*C-P & 0.04 & 2.22 & .03 & {$[0.00,0.07]$} & & \\
\hline \multirow{8}{*}{$\begin{array}{l}\text { Bayesian } \\
\text { surprise }\end{array}$} & & & & & & 0.01 & 0.16 \\
\hline & Bay. surprise & 0.04 & 3.21 & $<.01$ & {$[0.01,0.06]$} & & \\
\hline & block & 0.06 & 1.25 & .22 & {$[-0.04,0.17]$} & & \\
\hline & C-P & -0.11 & -2.07 & .04 & {$[-0.23,0.00]$} & & \\
\hline & Bay. surprise*block & -0.03 & -1.53 & .13 & {$[-0.06,0.01]$} & & \\
\hline & Bay. surprise*C-P & 0.00 & -0.17 & .86 & {$[-0.03,0.02]$} & & \\
\hline & Block*C-P & 0.01 & 0.21 & .84 & {$[-0.09,0.11]$} & & \\
\hline & $\begin{array}{l}\text { Bay. surprise*block* } \\
\text { C-P }\end{array}$ & 0.02 & 1.40 & .16 & {$[-0.01,0.06]$} & & \\
\hline
\end{tabular}

Notes. Coefficients of the fixed effects in the separate linear mixed-effects models of pupil dilation by entropy and Bayesian surprise (both z-scored per block and participant; model included nested random effects for block within participant), including C-P score as a predictor; block = contrast of the second, cued task block to the first, volatile task block; C-P = average score of the positive dimension scale of the CAPE (Community Assessment of Psychic Experiences); $R^{2} m=$ marginal $R^{2}$, i.e. proportion of 
variance explained by the fixed effects alone; $R^{2} c=$ conditional $R^{2}$, i.e. proportion of variance explained by both the fixed and random effects $\left(R^{2} m\right.$ and $R^{2} c$ based on Nakagawa \& Schielzeth, 2013). Results are rounded to two decimal places.

\section{Figure 5}

Pupil Responses to Entropy and Bayesian Surprise, Moderated by CAPE-P Scores
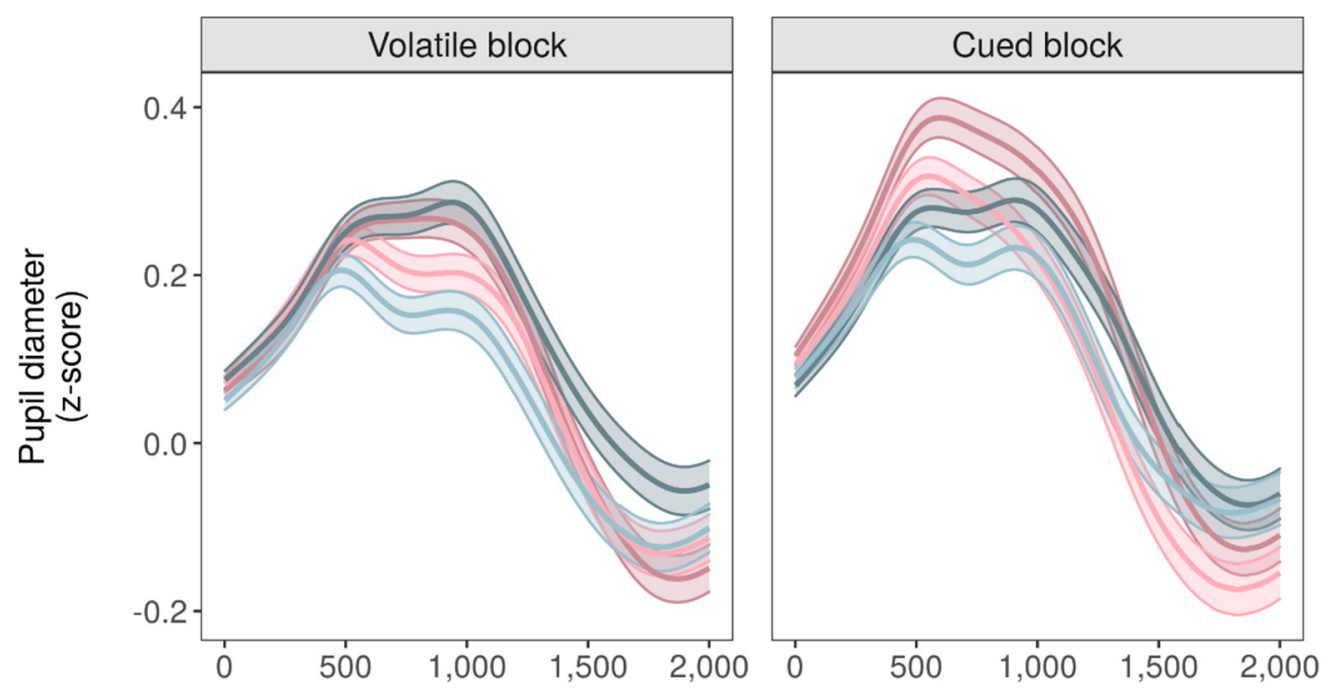

C-P score \& Entropy
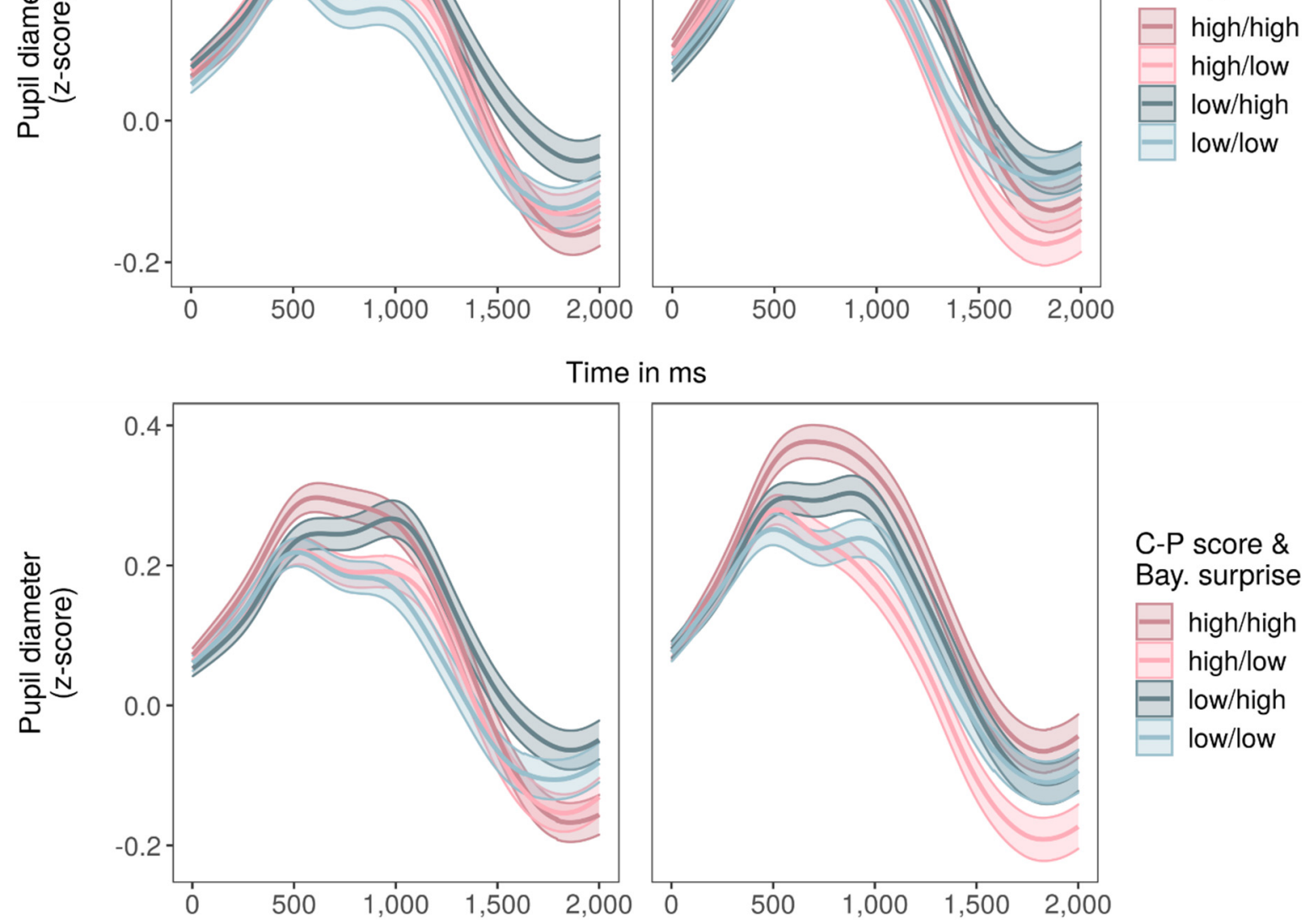

C-P score \&

Bay. surprise

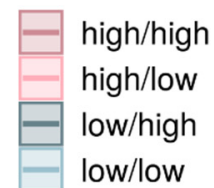

Time in ms

Notes. Pupil responses during outcome presentation to entropy (top row) and Bayesian surprise (bottom row) are presented separately for the two task blocks (columns). Colors differentiate between responses for trials with high or low entropy/Bayesian surprise (defined as values within participant-specific upper and lower quartile) and participants scoring high or low on the CAPE-P (defined as values above or 
below the sample-based median). Red-ish colors indicate a high, blue-ish colors a low CAPE-P score, darker shades represent high, brighter shades low entropy/Bayesian surprise values. Mean (solid line) and standard error of the mean (shaded area) were calculated for each sample of the z-scored and baseline-corrected pupil signal during outcome presentation. The quartile- and median-based categorizations were not used in any of the statistical models and only applied here to increase readability of the figure.

\section{Discussion}

Using a probabilistic reversal learning task and concurrent pupillometry, this study investigated the association of autistic-like traits (AQ) and psychotic-like experiences (CAPEP) with uncertainty processing in a sample of neurotypical adults. In contrast to findings from reversal learning studies using clinical samples (Culbreth et al., 2016; Deserno et al., 2020; Mussey et al., 2015; Waltz et al., 2013), the amount of switching per se was not significantly elevated in individuals with high AQ or CAPE-P scores. This may be due to lower symptom load in neurotypical samples as well as differences in task design (e.g., regarding the use of monetary rewards [Culbreth et al., 2016; Deserno et al., 2020; Mussey et al., 2015; Waltz et al., 2013] or different risk conditions [Culbreth et al., 2016; Deserno et al., 2020; Waltz et al., 2013]). Participants switched more often between the two choice options on high-risk compared to low-risk trials, possibly resulting from increased uncertainty about the favorable response as well as 'matching' behavior typical for probabilistic learning tasks (Feher da Silva et al., 2017). This effect was significantly smaller for participants with higher AQ scores, suggesting deficits in implicit probability learning (i.e., differentiating between risk conditions), in line with previous findings (Solomon et al., 2015). 
Computational modelling revealed that subjective volatility was significantly higher for participants scoring high on CAPE-P in the cued, low-volatile task block, replicating prior findings of volatility overestimation in psychotic disorders such as schizophrenia (Cole et al., 2020; Deserno et al., 2020). This may seem surprising, as changes were announced in this block. It is possible that, given higher initial volatility assumptions, these participants still perceived the states between announced changes as more unstable or that a failure to learn about the underlying risks and subsequently increased estimation uncertainty periodically caused more exploratory behavior, resulting in an increased volatility estimate. This uncertainty may have been too subtle to translate into behaviorally measured switching effects or general differences in choice uncertainty.

Although the absence of AQ or CAPE-score correlations with subjective volatility during the volatile task block implies no effect of trait and experience scores on volatility representation under conditions of high volatility, pupillometric findings point to differences with regards to how individuals react to this volatility. Overall, pupil dilation scaled positively with both entropy and Bayesian surprise. While Bayesian surprise signals the extent to which a belief should be updated, entropy expresses the uncertainty surrounding current beliefs. Hence, both relate to the informational value of the currently presented outcome for learning and belief updating. Their associations with pupil dilation replicate previous findings (Hämmerer et al., 2019; Kreis et al., 2021) and fit well with the assumption that pupil size as an indicator for LCNE activity signals neural gain and learning (Eldar et al., 2013; Joshi et al., 2016). Interestingly, there was a reduced effect of entropy on pupil dilation in individuals with higher CAPE-P scores within the volatile task block, indicating that participants with higher CAPE-P scores tracked uncertainty less reliably in terms of their psychophysiological response. This may reflect a hypersensitivity to volatility which increases the difficulty of keeping track of underlying uncertainty changes or decreases the subjective relevance of doing so. A similar pattern was 
found for individuals with higher AQ scores and Bayesian surprise, with a diminished differentiation between high and low surprise values in the volatile, and enhanced differentiation in the cued task block. The former again suggests hypersensitivity to volatile environments, where differentiation between stimuli that warrant a strong from those that warrant a weak (or no) belief update fails. The latter may be caused by an overestimation of volatility in an objectively rather stable environment where Bayesian surprise per se is smaller and belief updating in response to surprise signals should be less drastic than in stable environments. The positive correlation between AQ scores and subjective volatility in the cued task block, albeit not statistically significant, aligns with this interpretation.

These results fit well with previous findings of diminished pupil responses to surprise and uncertainty in individuals with autism (Lawson et al., 2017) and schizophrenia (Kreis et al., 2021). Notably, pupil responses to entropy and Bayesian surprise were affected differently by the different traits and experiences. This divergence may indicate that uncertaintyrepresentation related processes are more vulnerable to psychotic-like experiences and surpriserepresentation related processes more vulnerable to autistic-like traits, but this question warrants further investigation. Future studies should aim to include larger samples or preselect participants scoring particularly high and low on the AQ and the CAPE-P to include a wider range of trait and experience scores.

Together, these results provide important insights into how autistic- and psychotic-like traits and experiences are related to processing and representation of different kinds of uncertainty - even in neurotypical individuals. While psychotic-like experiences were associated with overestimation of volatility in a low-volatile period of the task, behavioral results further point to a link between autistic-like traits and risk misestimation. Psychophysiological results revealed a distinct pattern of abnormal neural gain adaptation to uncertainty and surprise for psychotic- and autistic-like traits and experiences, respectively, 
particularly when environmental volatility is high. This is in line with theoretical accounts of abnormal uncertainty processing and consequentially aberrant belief updating in psychosis and autism spectrum disorders. 


\section{References}

Abu-Akel, A. M., Wood, S. J., Hansen, P. C., \& Apperly, I. A. (2015). Perspective-taking abilities in the balance between autism tendencies and psychosis proneness. Proc Biol Sci, 282(1808), 20150563. https://doi.org/10.1098/rspb.2015.0563

Adams, R. A., Stephan, K. E., Brown, H. R., Frith, C. D., \& Friston, K. J. (2013). The computational anatomy of psychosis. Front Psychiatry, 4, 47. https://doi.org/10.3389/fpsyt.2013.00047

Ahn, W. Y., Haines, N., \& Zhang, L. (2017). Revealing Neurocomputational Mechanisms of Reinforcement Learning and Decision-Making With the hBayesDM Package. Comput Psychiatr, 1, 24-57. https://doi.org/10.1162/CPSY_a_00002

Aston-Jones, G., \& Cohen, J. D. (2005). An integrative theory of locus coeruleusnorepinephrine function: adaptive gain and optimal performance. Annu Rev Neurosci, 28, 403-450. https://doi.org/10.1146/annurev.neuro.28.061604.135709

Bevan Jones, R., Thapar, A., Lewis, G., \& Zammit, S. (2012). The association between early autistic traits and psychotic experiences in adolescence. Schizophrenia Research, 135(1), 164-169. https://doi.org/https://doi.org/10.1016/j.schres.2011.11.037

Browning, M., Behrens, T. E., Jocham, G., O'Reilly, J. X., \& Bishop, S. J. (2015). Anxious individuals have difficulty learning the causal statistics of aversive environments. Nat Neurosci, 18(4), 590-596. https://doi.org/10.1038/nn.3961

Cole, D. M., Diaconescu, A. O., Pfeiffer, U. J., Brodersen, K. H., Mathys, C. D., Julkowski, D., ... Stephan, K. E. (2020). Atypical processing of uncertainty in individuals at risk for

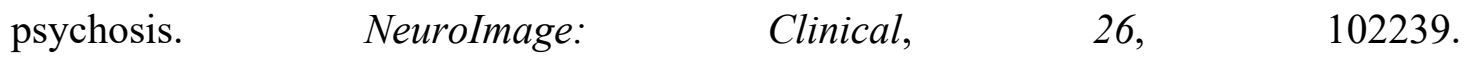
https://doi.org/https://doi.org/10.1016/j.nicl.2020.102239 
Culbreth, A. J., Gold, J. M., Cools, R., \& Barch, D. M. (2016). Impaired Activation in Cognitive Control Regions Predicts Reversal Learning in Schizophrenia. Schizophrenia Bulletin, 42(2), 484-493. https://doi.org/10.1093/schbul/sbv075

D'Cruz, A.-M., Ragozzino, M. E., Mosconi, M. W., Shrestha, S., Cook, E. H., \& Sweeney, J. A. (2013). Reduced behavioral flexibility in autism spectrum disorders. Neuropsychology, 27(2), 152-160. https://doi.org/10.1037/a0031721

de Berker, A. O., Rutledge, R. B., Mathys, C., Marshall, L., Cross, G. F., Dolan, R. J., \& Bestmann, S. (2016). Computations of uncertainty mediate acute stress responses in humans. Nat Commun, 7, 10996. https://doi.org/10.1038/ncomms10996

den Ouden, Hanneke E. M., Daw, Nathaniel D., Fernandez, G., Elshout, Joris A., Rijpkema, M., Hoogman, M., . . Cools, R. (2013). Dissociable Effects of Dopamine and Serotonin on Reversal Learning. $\quad$ Neuron, $\quad$ 1090-1100. https://doi.org/https://doi.org/10.1016/j.neuron.2013.08.030

Deserno, L., Boehme, R., Mathys, C., Katthagen, T., Kaminski, J., Stephan, K. E., . . . Schlagenhauf, F. (2020). Volatility Estimates Increase Choice Switching and Relate to Prefrontal Activity in Schizophrenia. Biological Psychiatry: Cognitive Neuroscience and Neuroimaging, $5(2)$, 173-183. https://doi.org/https://doi.org/10.1016/j.bpsc.2019.10.007

Eldar, E., Cohen, J. D., \& Niv, Y. (2013). The effects of neural gain on attention and learning. Nat Neurosci, 16(8), 1146-1153. https://doi.org/10.1038/nn.3428

Feher da Silva, C., Victorino, C. G., Caticha, N., \& Baldo, M. V. C. (2017). Exploration and recency as the main proximate causes of probability matching: a reinforcement learning analysis. Scientific Reports, 7(1), 15326. https://doi.org/10.1038/s41598-017-15587-z 
Fletcher, P. C., \& Frith, C. D. (2009). Perceiving is believing: a Bayesian approach to explaining the positive symptoms of schizophrenia. Nat Rev Neurosci, 10(1), 48-58. https://doi.org/10.1038/nrn2536

Friston, K., Kilner, J., \& Harrison, L. (2006). A free energy principle for the brain. Journal of Physiology-Paris, $100(1)$ $70-87$. https://doi.org/https://doi.org/10.1016/j.jphysparis.2006.10.001

Gelman, A., Carlin, J. B., Stern, H. S., Dunson, D. B., Vehtari, A., \& Rubin, D. B. (2013). Bayesian data analysis. CRC press.

Gläscher, J., Hampton, A. N., \& O'Doherty, J. P. (2008). Determining a Role for Ventromedial Prefrontal Cortex in Encoding Action-Based Value Signals During Reward-Related Decision Making. Cerebral Cortex, 19(2), 483-495. https://doi.org/10.1093/cercor/bhn098

Goris, J., Silvetti, M., Verguts, T., Wiersema, J. R., Brass, M., \& Braem, S. (2020). Autistic traits are related to worse performance in a volatile reward learning task despite adaptive $\begin{array}{lll}\text { learning } & \text { rates. } & \text { Autism, }\end{array}$ https://doi.org/10.1177/1362361320962237

Hämmerer, D., Schwartenbeck, P., Gallagher, M., FitzGerald, T. H. B., Düzel, E., \& Dolan, R. J. (2019). Older adults fail to form stable task representations during model-based reversal inference. Neurobiology of Aging, 74, 90-100. https://doi.org/https://doi.org/10.1016/j.neurobiolaging.2018.10.009

Hoekstra, R. A., Vinkhuyzen, A. A., Wheelwright, S., Bartels, M., Boomsma, D. I., BaronCohen, S., . . van der Sluis, S. (2011). The construction and validation of an abridged version of the autism-spectrum quotient (AQ-Short). J Autism Dev Disord, 41(5), 589596. https://doi.org/10.1007/s10803-010-1073-0 
Joshi, S., Li, Y., Kalwani, R. M., \& Gold, J. I. (2016). Relationships between Pupil Diameter and Neuronal Activity in the Locus Coeruleus, Colliculi, and Cingulate Cortex. Neuron, 89(1), 221-234. https://doi.org/10.1016/j.neuron.2015.11.028

Kaplan, C. M., Saha, D., Molina, J. L., Hockeimer, W. D., Postell, E. M., Apud, J. A., . . Tan, H. Y. (2016). Estimating changing contexts in schizophrenia. Brain, 139(7), 2082-2095. https://doi.org/10.1093/brain/aww095

Klingner, J., Kumar, R., \& Hanrahan, P. (2008). Measuring the task-evoked pupillary response with a remote eye tracker. Proceedings of the 2008 symposium on Eye tracking research $\&$ applications,

Kreis, I., Zhang, L., Moritz, S., \& Pfuhl, G. (2021). Spared performance but increased uncertainty in schizophrenia: Evidence from a probabilistic decision-making task. Schizophrenia Research. https://doi.org/https://doi.org/10.1016/j.schres.2021.06.038

Lawson, R. P., Mathys, C., \& Rees, G. (2017). Adults with autism overestimate the volatility of the sensory environment. Nat Neurosci, 20(9), 1293-1299. https://doi.org/10.1038/nn.4615

Lawson, R. P., Rees, G., \& Friston, K. J. (2014). An aberrant precision account of autism [Hypothesis and Theory]. Frontiers in Human Neuroscience, 8(302). https://doi.org/10.3389/fnhum.2014.00302

Li, C. T., Lai, W. S., Liu, C. M., \& Hsu, Y. F. (2014). Inferring reward prediction errors in patients with schizophrenia: a dynamic reward task for reinforcement learning. Front Psychol, 5, 1282. https://doi.org/10.3389/fpsyg.2014.01282

Martinez, A. P., Wickham, S., Rowse, G., Milne, E., \& Bentall, R. P. (2020). Robust association between autistic traits and psychotic-like experiences in the adult general population: epidemiological study from the 2007 Adult Psychiatric Morbidity Survey and 
replication with the 2014 APMS. Psychological Medicine, 1-7. https://doi.org/10.1017/s0033291720001373

Mathôt, S., Fabius, J., Van Heusden, E., \& Van der Stigchel, S. (2018). Safe and sensible preprocessing and baseline correction of pupil-size data [journal article]. Behavior Research Methods, 50(1), 94-106. https://doi.org/10.3758/s13428-017-1007-2

Murray, G. K., Cheng, F., Clark, L., Barnett, J. H., Blackwell, A. D., Fletcher, P. C., . . Jones, P. B. (2008). Reinforcement and reversal learning in first-episode psychosis. Schizophrenia Bulletin, 34(5), 848-855. https://doi.org/10.1093/schbul/sbn078

Mussey, J. L., Travers, B. G., Klinger, L. G., \& Klinger, M. R. (2015). Decision-Making Skills in ASD: Performance on the Iowa Gambling Task. Autism Research, 8(1), 105-114. https://doi.org/10.1002/aur.1429

Naicker, P., Anoopkumar-Dukie, S., Grant, G. D., Neumann, D. L., \& Kavanagh, J. J. (2016). Central cholinergic pathway involvement in the regulation of pupil diameter, blink rate and cognitive function. Neuroscience, 334, 180-190. https://doi.org/https://doi.org/10.1016/j.neuroscience.2016.08.009

Nakagawa, S., \& Schielzeth, H. (2013). A general and simple method for obtaining R2 from generalized linear mixed-effects models. Methods in Ecology and Evolution, 4(2), 133142. https://doi.org/10.1111/j.2041-210x.2012.00261.x

Nassar, M. R., Rumsey, K. M., Wilson, R. C., Parikh, K., Heasly, B., \& Gold, J. I. (2012). Rational regulation of learning dynamics by pupil-linked arousal systems. Nat Neurosci, 15(7), 1040-1046. https://doi.org/10.1038/nn.3130

Payzan-LeNestour, E., \& Bossaerts, P. (2011). Risk, unexpected uncertainty, and estimation uncertainty: Bayesian learning in unstable settings. PLoS Comput Biol, 7(1), e1001048. https://doi.org/10.1371/journal.pcbi.1001048 
Pearce, J. M., \& Hall, G. (1980). A model for Pavlovian learning: Variations in the effectiveness of conditioned but not of unconditioned stimuli. Psychological Review, 87(6), 532-552. https://doi.org/10.1037/0033-295X.87.6.532

Peirce, J., Gray, J. R., Simpson, S., MacAskill, M., Höchenberger, R., Sogo, H., . . Lindeløv, J. K. (2019). PsychoPy2: Experiments in behavior made easy. Behav Res Methods, 51(1), 195-203. https://doi.org/10.3758/s13428-018-01193-y

Pinheiro, J., Bates, D., DebRoy, S., Sarkar, D., R Core Team. (2021). nlme: Linear and Nonlinear Mixed Effects Models. R package version 3.1-152. https://CRAN.Rproject.org/package $=$ nlme

Powers, A. R., Mathys, C., \& Corlett, P. R. (2017). Pavlovian conditioning-induced hallucinations result from overweighting of perceptual priors. Science, 357(6351), 596600. https://doi.org/10.1126/science.aan3458

Preuschoff, K., t Hart, B. M., \& Einhauser, W. (2011). Pupil Dilation Signals Surprise: Evidence for Noradrenaline's Role in Decision Making. Front Neurosci, 5, 115. https://doi.org/10.3389/fnins.2011.00115

Rajkowski, J., Kubiak, P., \& Aston-Jones, G. (1994). Locus coeruleus activity in monkey: Phasic and tonic changes are associated with altered vigilance. Brain Research Bulletin, 35(5), 607-616. https://doi.org/https://doi.org/10.1016/0361-9230(94)90175-9

R Core Team. (2018). R: A language and environment for statistical computing. Version 3.5.1. https://www.r-project.org/

Rescorla, R. A., \& Wagner, A. R. (1972). A theory of Pavlovian conditioning: Variations in the effectiveness of reinforcement and nonreinforcement. In A. H. Black \& W. F. Prokasy (Eds.), Classical conditioning II: Current research and theory (pp. 64-99). Appleton Century Crofts. 
Robic, S., Sonié, S., Fonlupt, P., Henaff, M. A., Touil, N., Coricelli, G., . . Schmitz, C. (2015). Decision-making in a changing world: a study in autism spectrum disorders. $J$ Autism Dev Disord, 45(6), 1603-1613. https://doi.org/10.1007/s10803-014-2311-7

Samuels, E. R., \& Szabadi, E. (2008). Functional neuroanatomy of the noradrenergic locus coeruleus: its roles in the regulation of arousal and autonomic function part II: physiological and pharmacological manipulations and pathological alterations of locus coeruleus activity in humans. Current neuropharmacology, 6(3), 254-285. https://doi.org/10.2174/157015908785777193

Schlagenhauf, F., Huys, Q. J. M., Deserno, L., Rapp, M. A., Beck, A., Heinze, H.-J., . . Heinz, A. (2014). Striatal dysfunction during reversal learning in unmedicated schizophrenia patients. NeuroImage,

89 , 171-180. https://doi.org/https://doi.org/10.1016/j.neuroimage.2013.11.034

Solomon, M., Frank, M. J., Ragland, J. D., Smith, A. C., Niendam, T. A., Lesh, T. A., . . . Carter, C. S. (2015). Feedback-driven trial-by-trial learning in autism spectrum disorders. American Journal of Psychiatry, 172(2), 173-181. https://doi.org/https://doi.org/10.1176/appi.ajp.2014.14010036

Solomon, M., Smith, A. C., Frank, M. J., Ly, S., \& Carter, C. S. (2011). Probabilistic reinforcement learning in adults with autism spectrum disorders. Autism Research, 4(2), 109-120. https://doi.org/10.1002/aur.177

Stefanis, N. C., Hanssen, M., Smirnis, N. K., Avramopoulos, D. A., Evdokimidis, I. K., Stefanis, C. N., .. . Van Os, J. (2002). Evidence that three dimensions of psychosis have a distribution in the general population. Psychological Medicine, 32(2), 347-358. https://doi.org/10.1017/S0033291701005141

Steinhauer, S., \& Zubin, J. (1982). Vulnerability to schziophrenia: Information processing in the pupil and event-related potential. In E. Usdin \& I. Hanin (Eds.), Biological Markers 
in Psychiatry and Neurology (pp. 371-385). Pergamon. https://doi.org/https://doi.org/10.1016/B978-0-08-027987-9.50042-1

Steinhauer, S. R., Hakerem, G., \& Spring, B. J. (1979). The pupillary response as a potential indicator of vulnerability to schizophrenia. Psychopharmacology Bulletin, 15(1), 44-45.

Stone, J. M., \& Towse, J. N. (2015). A working memory test battery: Java-based collection of seven working memory tasks. . Journal of Open Research Software, 3(1), e5. https://doi.org/http://doi.org/10.5334/jors.br

Van de Cruys, S., Evers, K., Van der Hallen, R., Van Eylen, L., Boets, B., de-Wit, L., \& Wagemans, J. (2014). Precise minds in uncertain worlds: predictive coding in autism. Psychol Rev, 121(4), 649-675. https://doi.org/10.1037/a0037665

van Schalkwyk, G. I., Volkmar, F. R., \& Corlett, P. R. (2017). A predictive coding account of psychotic symptoms in autism spectrum disorder [Journal; Peer Reviewed Journal]. Journal of Autism and Developmental Disorders, 47(5), 1323-1340. https://doi.org/http://dx.doi.org/10.1007/s10803-017-3065-9 28185044

Waltz, J. A., \& Gold, J. M. (2007). Probabilistic reversal learning impairments in schizophrenia: Further evidence of orbitofrontal dysfunction. Schizophrenia Research, 93(1), 296-303. https://doi.org/https://doi.org/10.1016/j.schres.2007.03.010

Waltz, J. A., Kasanova, Z., Ross, T. J., Salmeron, B. J., McMahon, R. P., Gold, J. M., \& Stein, E. A. (2013). The Roles of Reward, Default, and Executive Control Networks in SetShifting Impairments in Schizophrenia. PLoS One, 8(2), e57257. https://doi.org/10.1371/journal.pone.0057257

Weickert, T. W., Goldberg, T. E., Egan, M. F., Apud, J. A., Meeter, M., Myers, C. E., .. . Weinberger, D. R. (2010). Relative risk of probabilistic category learning deficits in patients with schizophrenia and their siblings. Biol Psychiatry, 67(10), 948-955. https://doi.org/10.1016/j.biopsych.2009.12.027 
Wickham, H. (2016). ggplot2: Elegant Graphics for Data Analysis. Springer.

Worthy, D. A., \& Todd Maddox, W. (2014). A comparison model of reinforcement-learning and win-stay-lose-shift decision-making processes: A tribute to W.K. Estes. Journal of $\begin{array}{llll}\text { Mathematical } & \text { Psychology, } & 59, & \text { 41-49. }\end{array}$ https://doi.org/https://doi.org/10.1016/j.jmp.2013.10.001

Yu, A. J., \& Dayan, P. (2005). Uncertainty, Neuromodulation, and Attention. Neuron, 46(4), 681-692. https://doi.org/https://doi.org/10.1016/j.neuron.2005.04.026

Yung, A. R., Nelson, B., Baker, K., Buckby, J. A., Baksheev, G., \& Cosgrave, E. M. (2009). Psychotic-Like Experiences in a Community Sample of Adolescents: Implications for the Continuum Model of Psychosis and Prediction of Schizophrenia. Australian \& New Zealand Journal of Psychiatry, 43(2), 118-128. https://doi.org/10.1080/00048670802607188

Zhang, L., Lengersdorff, L., Mikus, N., Gläscher, J., \& Lamm, C. (2020). Using reinforcement learning models in social neuroscience: frameworks, pitfalls and suggestions of best practices. Social Cognitive and Affective Neuroscience. https://doi.org/10.1093/scan/nsaa089 


\title{
Supplementary Information for:
}

\section{Aberrant Uncertainty Processing Is Linked to Psychotic-like Experiences, Autistic Traits and Reflected in Pupil Dilation During Probabilistic Learning}

\author{
Isabel V. Kreis \\ Department of Psychology, UiT - The Arctic University of Norway, Tromsø, Norway \\ Lei Zhang \\ Department of Psychology, UiT - The Arctic University of Norway
}

Social, Cognitive and Affective Neuroscience Unit, Department of Cognition, Emotion, and Methods in Psychology, Faculty of Psychology, University of Vienna, Vienna,

Austria

Matthias Mittner

Department of Psychology, UiT - The Arctic University of Norway, Tromsø, Norway Leonard Syla

Department of Psychology, UiT - The Arctic University of Norway, Tromsø, Norway Claus Lamm

Social, Cognitive and Affective Neuroscience Unit, Department of Cognition, Emotion, and Methods in Psychology, Faculty of Psychology, University of Vienna, Vienna,

Austria

Vienna Cognitive Science Hub, University of Vienna, Vienna, Austria

Gerit Pfuhl

Department of Psychology, UiT - The Arctic University of Norway, Tromsø, Norway 


\section{Supplementary Methods: Working Memory Task}

Working memory capacity was assessed with the visual digit span and the matrix span task of a computerized open source working memory test battery (Stone \& Towse, 2015). On each trial of the digit span task, participants were presented with several digits between 10 and 99, one after another, and had to recall them in the correct order. The number of digits to recall (i.e. task load) increased by one after every third trial, ranging from two to seven. The maximum number of digits recalled in the correct order was used as an index for working memory capacity in the verbal-numerical domain. Load conditions and general structure were identical for the matrix span task, where instead of digits, indicated grid locations in a $4 \times 4$ grid had to be recalled. Here, maximum number of locations recalled in the correct order was used as an index for working memory capacity in the visual-spatial domain.

\section{Supplementary Methods: Computational Models}

Computation models were fitted to participants' choice data for the cued and the volatile block of the prediction task, respectively. The models included a simple win-stay-looseshift model (WSLS), four different Reinforcement Learning models, and two variants of a Hidden Markov Model.

All models are based on relating participant's choices with their subsequent outcome in one way or another. For the prediction task, those choices describe the prediction of either the left or the right tilted Gabor patch on a given trial. The outcome was then simply the observation of either the left or the right tilted patch, following the prediction. A positive feedback was defined as a match between prediction and outcome and assigned the reward value +1 . A negative feedback was in turn a mismatch between 
prediction and outcome and assigned the 'reward' value -1 (also referred to as 'punishment').

\section{Choice of models}

The WSLS model (Worthy \& Todd Maddox, 2014) assumes that participants continue to choose one of the two choice options (left/right) if they were rewarded for this choice on the previous trial but shift to the other option when they were not. It was included to allow for the fact that participants might employ a very simplistic prediction strategy, basing their choices purely on the outcome of the previous trial. The four RL models assume a more long-term integration of prediction errors and prediction values of the two choice options. The four versions differed in how this information was integrated. In the simplest Rescorla-Wagner model (RLRW; Rescorla \& Wagner, 1972), values for only the chosen action (i.e. predict the left or the right-tilted Gabor patch) were updated via learning rate weighted prediction errors. The reward-punishment model (RLRP; den Ouden et al., 2013) introduces different learning rates for positive (prediction correct) vs. negative feedback (prediction incorrect), since participants might respond differently to those events. Given the anti-correlated task structure, participants might learn about the values of the two choice options concurrently. This was accounted for in a counterfactual updating model (RLCF; Gläscher et al., 2008), where values for both actions were updated simultaneously. In an additional model based on Pearce and Hall (RLpH; Pearce \& Hall, 1980), the learning rate was adaptive and varied across the course of the respective task block. This could capture the fact that the weight assigned to a prediction error, and thus the extent of value updating, might change throughout the task and be larger after change points. The Hidden Markov Models (HMM; Schlagenhauf et al., 2014) were included to allow for a more strategic decision-making process that rests on a higher-level representation 
of the different task-states. They assume that participants choose between 'left' or 'right' based on their belief of being in a state of the task where either the left- or the right-tilted Gabor patch is more common, and on their estimated probability for the two states to change. To account for the fact that participants may be more or less sensitive to positive (prediction correct) vs. negative feedback (prediction incorrect), one HMM variant allowed for the effects of those outcomes to differ (HMMRP), and one assumed identical effects of positive and negative feedback (HMM). All models are described in detail below $(\mathrm{I}-\mathrm{V})$.

For the cued task block, additional variants of all models were specified that implemented a reset of beliefs about choice values and current state probabilities at each time point where participants were informed about a change in the underlying stimulus distribution. It was expected that after each of these announced change points, beliefs would be reset to their initial values $(0.5)$.

\section{(I) Win-Stay-Loose-Shift model}

As the first and simplest model, a Win-Stay-Lose-Shift model was fitted (WSLS; Worthy \& Todd Maddox, 2014). This model assumes that participants continue to choose one of the two available options (left/right) if they were rewarded for this choice on the previous trial ('win-stay'; i.e. their prediction turned out to be correct). Likewise, participants are assumed to pick the other option if they were not rewarded ('lose-shift'; i.e. their prediction turned out to be incorrect). Let $\mathrm{A}$ and $\mathrm{B}$ be the two actions, the value of 'staying' with the same choice option after a 'win' (meaning the net gain on the current trial is equal to or greater than zero) is then calculated as:

$$
\begin{gathered}
V(A \mid A, \text { win })=1 \\
V(B \mid A, \text { win })=-1
\end{gathered}
$$


Similarly, the value of 'switching' to the other option after a 'loss' (meaning the net gain on the current trial is less than zero) is:

$$
\begin{gathered}
V(B \mid A, \text { loss })=1 \\
V(A \mid A, \text { loss })=-1
\end{gathered}
$$

\section{(II) Standard Rescorla-Wagner model}

The second model was a standard Rescorla-Wagner reinforcement learning (RLRw; Rescorla \& Wagner, 1972) model with a constant learning rate and value updates for only the choses action. The updating rule of this model defines that for each trial $t$, the value $V$ of the current choice depends on the value and the prediction error $\delta$ (the difference between reward and expected value) of the previous trial $t-1$, weighted by the learning rate $\alpha$ :

$$
\mathrm{V}_{\mathrm{t}}=\mathrm{V}_{\mathrm{t}-1}+\alpha \times \delta
$$

Here, $\delta$ is the prediction error, calculated as:

$$
\delta=\left(\mathrm{R}_{\mathrm{t}-1}-\mathrm{V}_{\mathrm{t}-1}\right)
$$

To account for the possibility that the effects of 'rewards' (i.e. correct predictions) and 'punishments' (i.e. incorrect predictions) on learning might differ, an variant of the model was fitted with separate learning rates $\alpha$ for rewards and punishments (model: RLRP; den Ouden et al., 2013).

\section{(III) Counterfactual Reinforcement Learning model}

The third model was a counterfactual updating model (RLCF; Gläscher et al., 2008) that only differed from (II) in the way that values of both the chosen and the unchosen options were updated in parallel. The formula for the value update was the same as above but for the unchosen option (uc), a fictitious prediction error was used: 


$$
\delta^{\mathrm{f}}=\left(-\mathrm{R}_{\mathrm{t}-1}-\mathrm{V}_{\mathrm{t}-1}^{\mathrm{uc}}\right)
$$

\section{(IV) Pearce-Hall model}

The fourth model was a Pearce-hall model with a dynamic learning rate (RLPH; Pearce \& Hall, 1980). While value update was similar to the standard RLRW model (see II), the learning rate $\alpha$ varied across trials, updated from the previous prediction error:

$$
\alpha_{\mathrm{t}}=\gamma \times\left|\left(\mathrm{R}_{\mathrm{t}-1}-\mathrm{V}_{\mathrm{t}-1}\right)\right|+(1-\gamma) \times \alpha_{\mathrm{t}-1}
$$

\section{(V) Hidden Markov Model}

Lastly, behavior was modelled with a Hidden Markov Model (HMM; Schlagenhauf et al., 2014). Here, it is assumed that participants make their choices (i.e. predict either the left or the right Gabor patch) based on their belief about the current state of the task as there being either predominantly left ('state L') or predominantly right ('state R') tilted patches.

While inferring the belief distribution over the different states, participants are assumed to treat action-reward pairs (i.e. the combination of their prediction and the 'reward' in terms of the prediction being correct or incorrect) as observations: $O_{t}=$ $\left\{a_{t}, r_{t}\right\}$. A hidden state variable $S_{t}$ represents a participant's estimation of such an actionoutcome pair. The prior over the current state $P\left(S_{t} \mid S_{t-1}\right)$ is calculated as the posterior belief from the previous trial modulated by the reversal probability $\gamma$ (i.e., the transition probabilities between latent states) in a transition matrix:

$$
P\left(S_{t} \mid S_{t-1}\right)=\left(\begin{array}{lr}
1-\gamma & \gamma \\
\gamma & 1-\gamma
\end{array}\right)
$$

Here, $\gamma$ is a free parameter between 0 and 1 . 
The probability of observing the outcome corresponding to a given latent state depends on the probability $c$ with which a reward (i.e. positive feedback indicating a correct prediction) indicates that the true latent state indeed corresponds to the choice made and the probability $d$ with which a 'punishment' (i.e. negative feedback indicating an incorrect prediction) indicates that the latent state is not the one chosen by action $a$. It is updated as:

$$
P\left(O_{t} \mid S_{t}\right)=0.5 \times\left(\begin{array}{cr}
c & 1-c \\
1-d & d
\end{array}\right)
$$

$C$ and $d$ were treated as free parameters and initialized to lie between 0.5 and 1 . As in the RLRP model, this differentiation between 'rewards' and 'punishments' allowed for different effects of both in terms of their informativeness for model updating. This version of the model is here referred to as $\mathrm{HMM}_{\mathrm{RP}}$. For comparison, an additional version of the model was fitted where rewards and punishments were treated equally, with $c=d$. That version of the model is denoted as HMM.

On a given trial, the prior probability of $S_{t}$ before any outcome has been observed is calculated from the state transition probabilities (see above) and the posterior probability of $S_{t-1}$ :

$$
P\left(S_{t}\right)=\sum_{S_{t-1}} P\left(S_{t} \mid S_{t-1}\right) P\left(S_{t-1}\right)
$$

After outcome observation, the posterior probability of $S_{t}$ is then updated based on the prior $P\left(S_{t}\right)$ and the observed outcome $O_{t}$ :

$$
P\left(S_{t}\right)=\frac{P\left(O_{t} \mid S_{t}\right) P\left(S_{t}\right)}{\sum_{S_{t}} P\left(O_{t} \mid S_{t}\right) P\left(S_{t}\right)}
$$




\section{Softmax action selection}

For all models (I) - (IV), values were translated into choice probabilities for options $L$ and $R$ with a softmax action selector:

$$
p(R)=\frac{1}{1-e^{\beta \times\left(-\left(V_{R}-V_{L}\right)\right.}}, \quad p(L)=1-p(R)
$$

Here, $\beta$ is the inverse temperature, which determines the slope of the sigmoid function and the stochasticity (randomness) of the choices. For model (V), state probabilities, instead of values, were used in the softmax function. Also, to reduce non-identifiable parameter estimation, the inverse temperature parameter was not included for model (V).

\section{Model comparison}

All model parameters were estimated using Hierarchical Bayesian Analysis (HBA; Gelman et al., 2013) implemented in the Stan language in R (Stan Development Team, 2016), adopted from the hBayesDM package (Ahn et al., 2017). For both task blocks separately, models were compared regarding their goodness-of-fit to explain the observed data whilst accounting for model complexity. Model fit was compared using leave-one-out cross validation, calculated using the log-likelihood evaluated at the posterior simulations. Model fit was reported as leave-one-out information criterion (LOOIC), with lower LOOIC indicating better model fit. Model fit results are reported in Table S1 for the first, volatile block of the task and in Table S2 for the second, cued block of the task. The HMMRP provided the best fit for both the volatile and the cued task block. Notably, our group recently conducted a study using the same task paradigm and an identical set of models. Here, a model recovery analysis revealed that all candidate models could be properly identified and recovered (Kreis et al., 2021). 
Table S1

Task block 1 (volatile): Model fit

\begin{tabular}{ccc} 
Model & LOOIC & no. of parameters \\
\hline WSLS & 9950 & 1 \\
RLRW & 9761 & 2 \\
RLCF & 9505 & 2 \\
RLRP & 9717 & 3 \\
RLPH & 9756 & 4 \\
HMM & 9559 & 2 \\
HMM $_{\mathbf{R P}}$ & $\mathbf{9 3 9 1}$ & $\mathbf{3}$ \\
\hline
\end{tabular}

\section{Table S2}

Task block 2 (cued): Model fit

\begin{tabular}{|c|c|c|}
\hline Model & LOOIC & no. of parameters \\
\hline WSLS & 9982 & 1 \\
\hline RLRW & 9986 & 2 \\
\hline RLRW_reset & 9189 & 2 \\
\hline RLCF & 10248 & 2 \\
\hline RLCF_reset & 8897 & 2 \\
\hline RLRP & 10222 & 3 \\
\hline RLRP_reset & 8796 & 3 \\
\hline RLPH & 9981 & 4 \\
\hline RLPH_reset & 9189 & 4 \\
\hline HMM & 9448 & 2 \\
\hline $\mathrm{HMM}_{-}$reset & 8902 & 2 \\
\hline $\mathrm{HMM}_{\mathrm{RP}}$ & 9018 & 3 \\
\hline HMM $M_{\text {RP_reset }}$ & 8416 & 3 \\
\hline
\end{tabular}




\section{Supplementary Results}

Questionnaire scores (AQ and CAPE-P) are not related to demographic or working memory variables

Questionnaire scores were not related to age (AQ: $\rho=-0.13, p=.35$; CAPE-P: $\rho=-0.22$, $p=.11$ ), education (AQ: $\chi^{2}(2)=0.58, p=.75$; CAPE-P: $\left.\chi^{2}(2)=0.73, p=.69\right)$, gender (AQ: $\mathrm{W}=294.50, p=0.57$; CAPE-P: $\mathrm{W}=310.50, p=.79$ ), verbal-numerical (AQ: $\rho=$ $0.03, p=.85$; CAPE-P: $\rho=0.04, p=.78$ ) or visual-spatial working memory capacity (AQ: $\rho=0.03, p=.81$; CAPE-P: $\rho=0.22, p=.12$ ).

Entropy and Bayesian surprise differ by task conditions but not questionnaire scores

In two separate linear mixed-effects models, both entropy (choice uncertainty) and Bayesian surprise (belief updating) were significantly higher on high-risk trials (Table S3). For entropy, this effect was more pronounced within the cued task block (block*risk: $\beta=0.18, t=2.95, p<.01,95 \%$ CI $[0.06,0.31])$, where high- and low-risk conditions would be easier to distinguish due to the absence of hidden changes. Bayesian surprise was lower in the cued task block, indicating reduced belief updating under low volatility $(\beta=-0.40, t=-4.53, p<.001,[-0.58,-0.23])$. 


\section{Table S3}

Linear mixed-effects model results for latent $H M_{R P}$ variables by task conditions

\begin{tabular}{llllllll} 
DV & & $\beta$ & $t$ & $p$ & CI $(95 \%)$ & $R^{2}{ }_{M}$ & $R^{2} C$ \\
\hline Entropy & & & & & & 0.03 & 0.42 \\
& Block & -0.07 & -0.66 & .51 & {$[-0.28,0.14]$} & & \\
& Risk & 0.23 & 5.18 & $<.001$ & {$[0.14,0.32]$} & & \\
Bayesian surprise & Block*Risk & 0.18 & 2.95 & $<.01$ & {$[0.06,0.31]$} & & \\
& Block & -0.40 & -4.53 & $<.001$ & {$[-0.58,-0.23]$} & & \\
& Risk & 0.20 & 6.75 & $<.001$ & {$[0.14,0.26]$} & & \\
& Block*Risk & 0.01 & 0.12 & .90 & {$[-0.8,0.09]$} & & \\
\hline
\end{tabular}

Notes: Coefficients of the fixed effects in the linear mixed-effects models for the different dependent variables (DV); Entropy $=$ choice uncertainty $\left(\mathrm{HMM}_{\mathrm{RP}}\right)$; Bayesian surprise $=$ cube root transformed belief updating $\left(\mathrm{HMM}_{\mathrm{RP}}\right)$; Block = contrast of the second, cued task block to the first, volatile task block; Risk = contrast of the high- to the low-risk condition; $R^{2} m=$ marginal $R^{2}$, i.e. proportion of variance explained by the fixed effects alone; $R^{2} c=$ conditional $R^{2}$, i.e. proportion of variance explained by both the fixed and random effects $\left(R^{2} m\right.$ and $R^{2} c$ based on Nakagawa \& Schielzeth, 2013). Results are rounded to two decimal places.

Inclusion of AQ and CAPE-P scores, respectively, did not reveal any significant scorerelated main or interaction effects - neither in the entropy model (AQ-model: AQ: $\beta=$ $0.06, t=0.65, p=.52,95 \%$ CI $[-0.12,0.23]$; block*AQ: $\beta=0.06, t=0.54, p=.59,[-$ $0.15,0.27]$; $\operatorname{risk}^{*} \mathrm{AQ}: \beta=-0.02, t=-0.39, p=.69,[-0.10,0.07]$; block*risk*AQ: $\beta=$ 0.02, $t=0.29, p=.77,[-0.11,0.14]$; CAPE-P model: CAPE-P: $\beta=-0.05, t=-0.53, p=$ $.60,[-0.22,0.13]$; block*CAPE-P: $\beta=0.15, t=1.46, p=.15,[-0.06,0.36]$; risk*CAPE$\mathrm{P}: \beta=-0.03, t=-0.80, p=.43,[-0.12,0.05]$; block*risk*CAPE-P: $\beta=0.01, t=-0.12, p$ $=.91,[-0.13,0.12])$, nor the Bayesian surprise model (AQ-model: AQ: $\beta=0.05, t=0.60$, $p=.55,[-0.11,0.21]$; block*AQ: $\beta=0.02, t=0.27, p=.79,[-0.16,0.20]$; risk*AQ: $\beta=$ $-0.02, t=-0.81, p=.42,[-0.08,0.04] ;$ block* risk$^{*} \mathrm{AQ}: \beta=0.01, t=0.23, p=.82,[-0.07$, 0.09]; CAPE-P-model: CAPE-P: $\beta=0.13, t=1.63, p=.11,[-0.03,0.29]$; block*CAPE- 
$\mathrm{P}: \beta=0.02, t=0.24, p=.81,[-0.16,0.20]$; risk ${ }^{*} \mathrm{CAPE}-\mathrm{P}: \beta=-0.01, t=-0.36, p=.72,[-$ 0.07, 0.05]; block*risk*CAPE-P: $\beta=0.00, t=0.08, p=.94,[-0.08,0.09])$.

\section{Supplementary Figures: Interaction Effects}

Figures below display predicted values of the 3-way interaction terms from the linearmixed effects models of pupil size by task block, belief entropy/Bayesian surprise, and AQ/CAPE-P scores, respectively, plotted with the sjPlot package (version 2.8.9; Lüdecke, 2021). In each Figure, the continuous questionnaire score variables were grouped by minimum (blue color), median (purple color), and maximum value (red color) for demonstration purposes. Left panels present values for the first, volatile task block, and right panels values for the second, cued task block.

\section{Figure S1}

Predicted values of maximum pupil dilation by block, entropy, and AQ scores

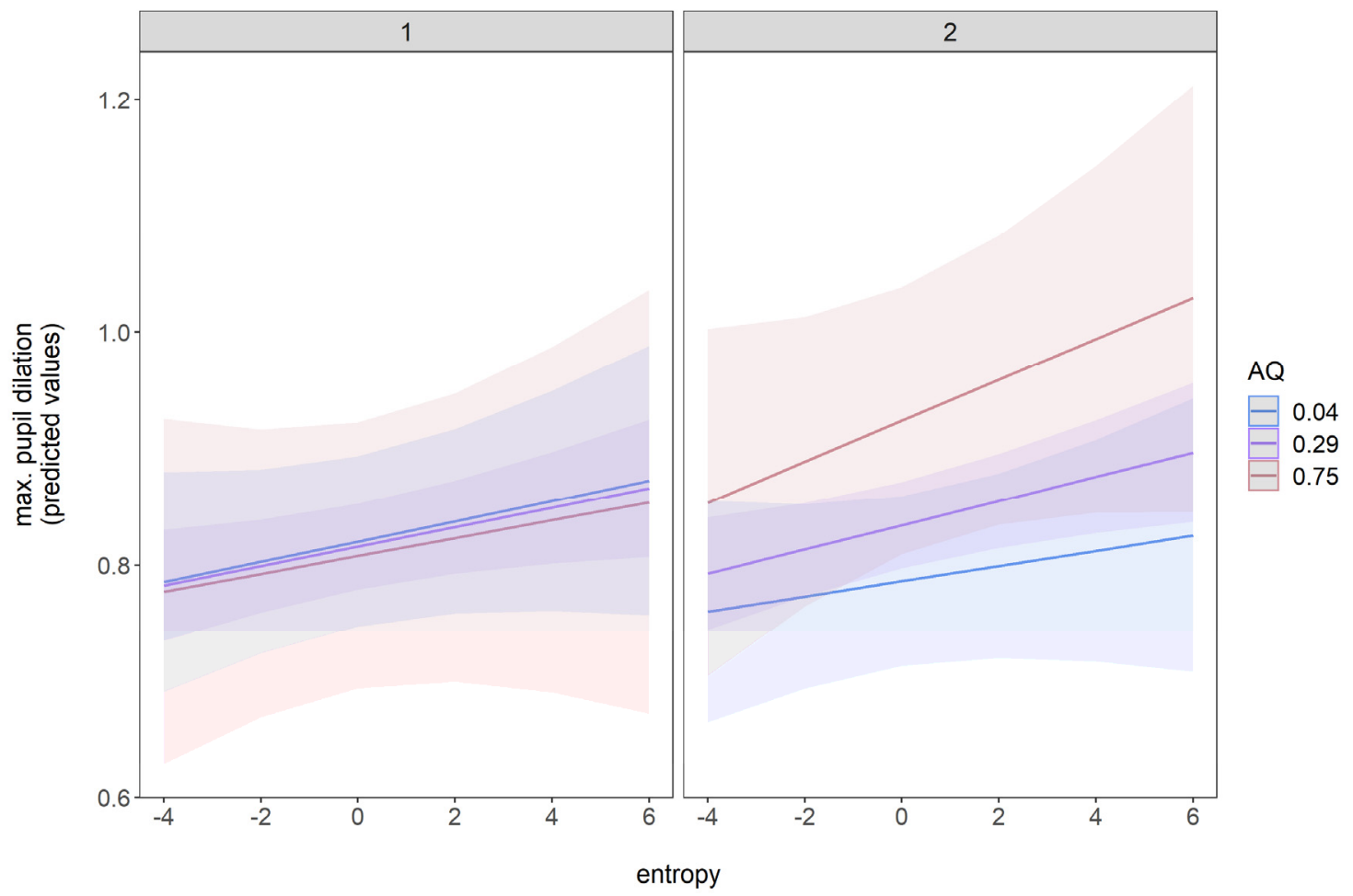




\section{Figure S2}

Predicted values of maximum pupil dilation by block, Bayesian surprise, and AQ scores.

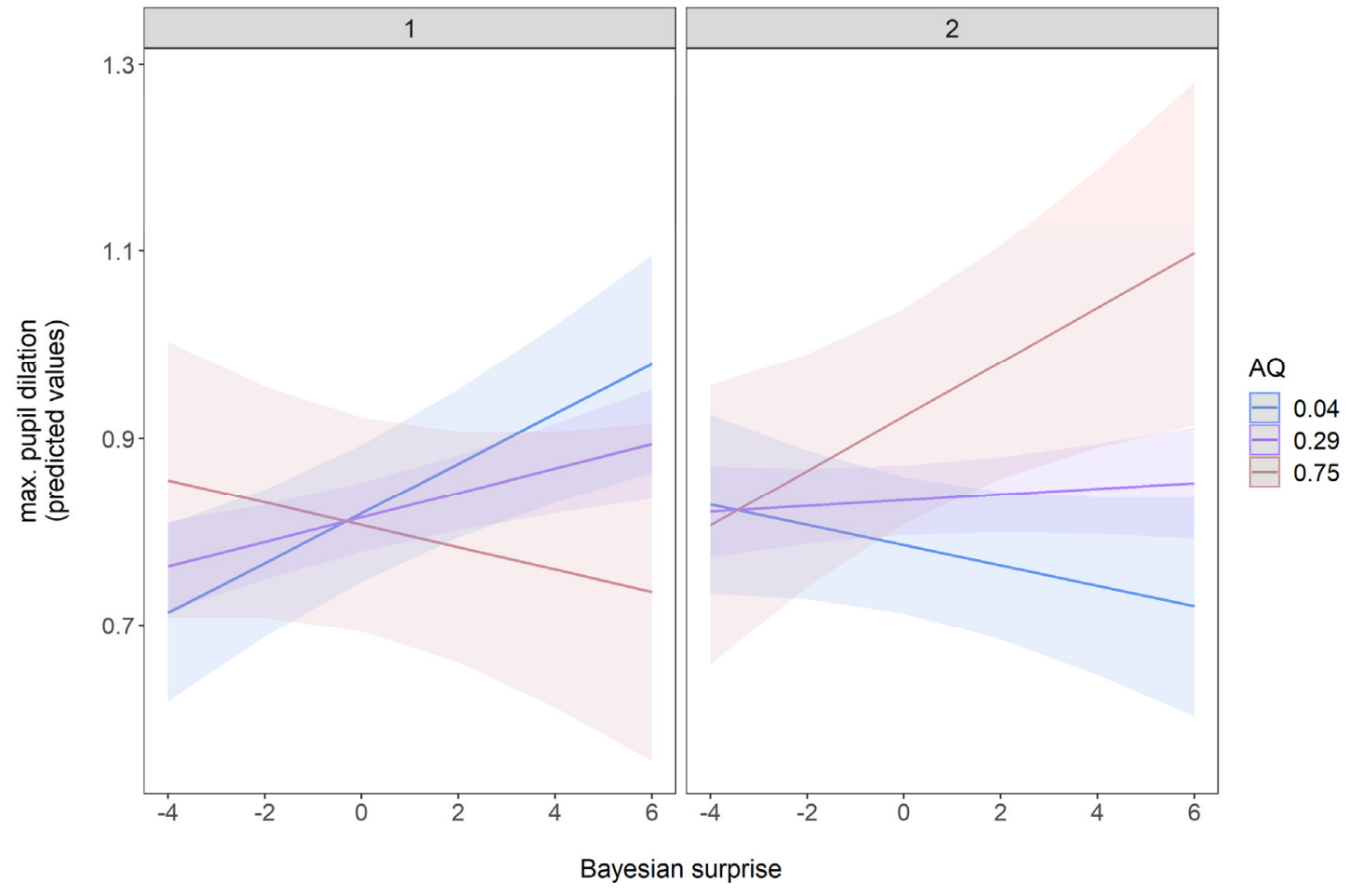

\section{Figure S3}

Predicted values of maximum pupil dilation by block, entropy, and CAPE-P scores

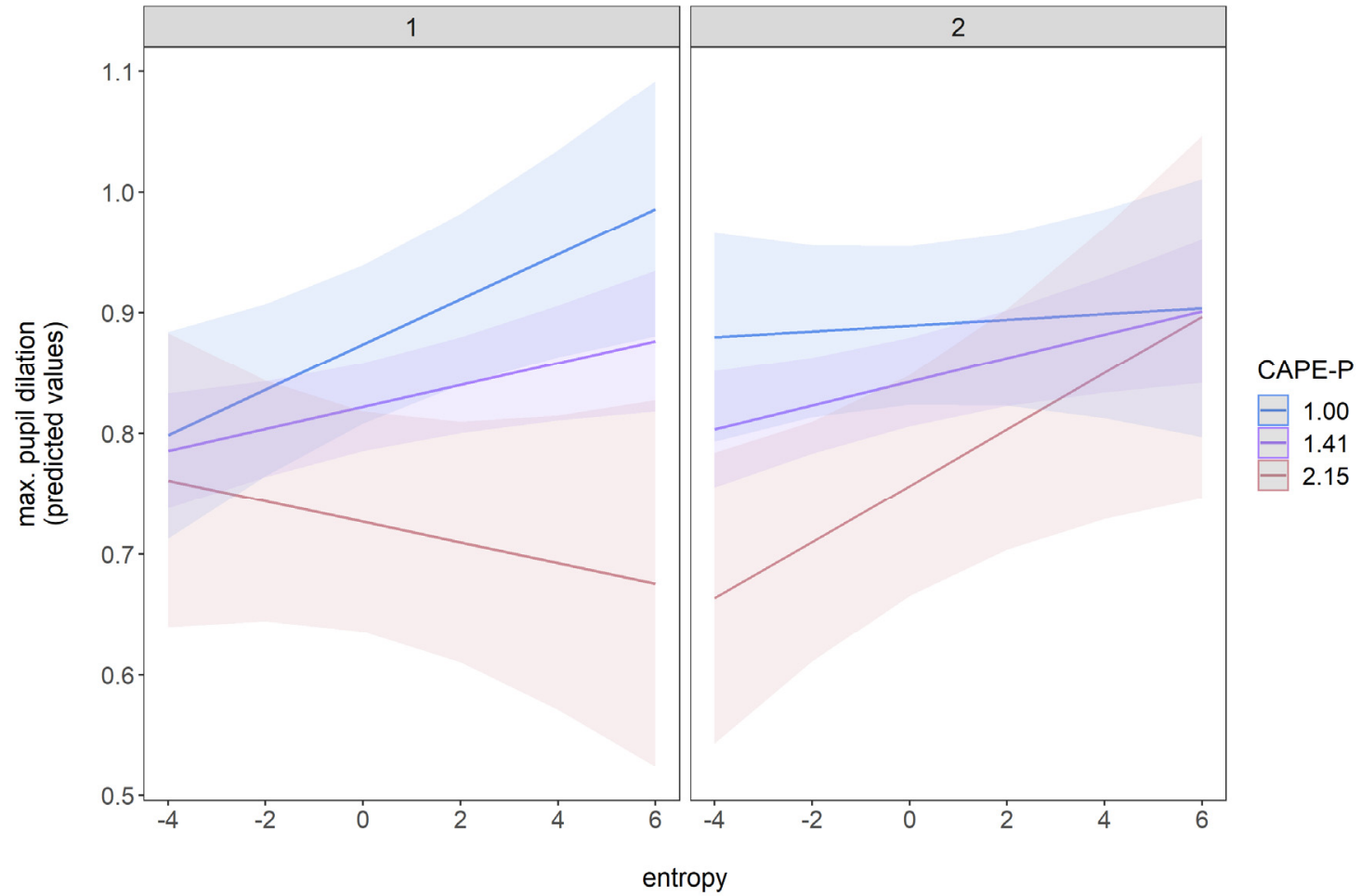




\section{Figure S4}

Predicted values of maximum pupil dilation by block, Bayesian surprise, and CAPE-P scores

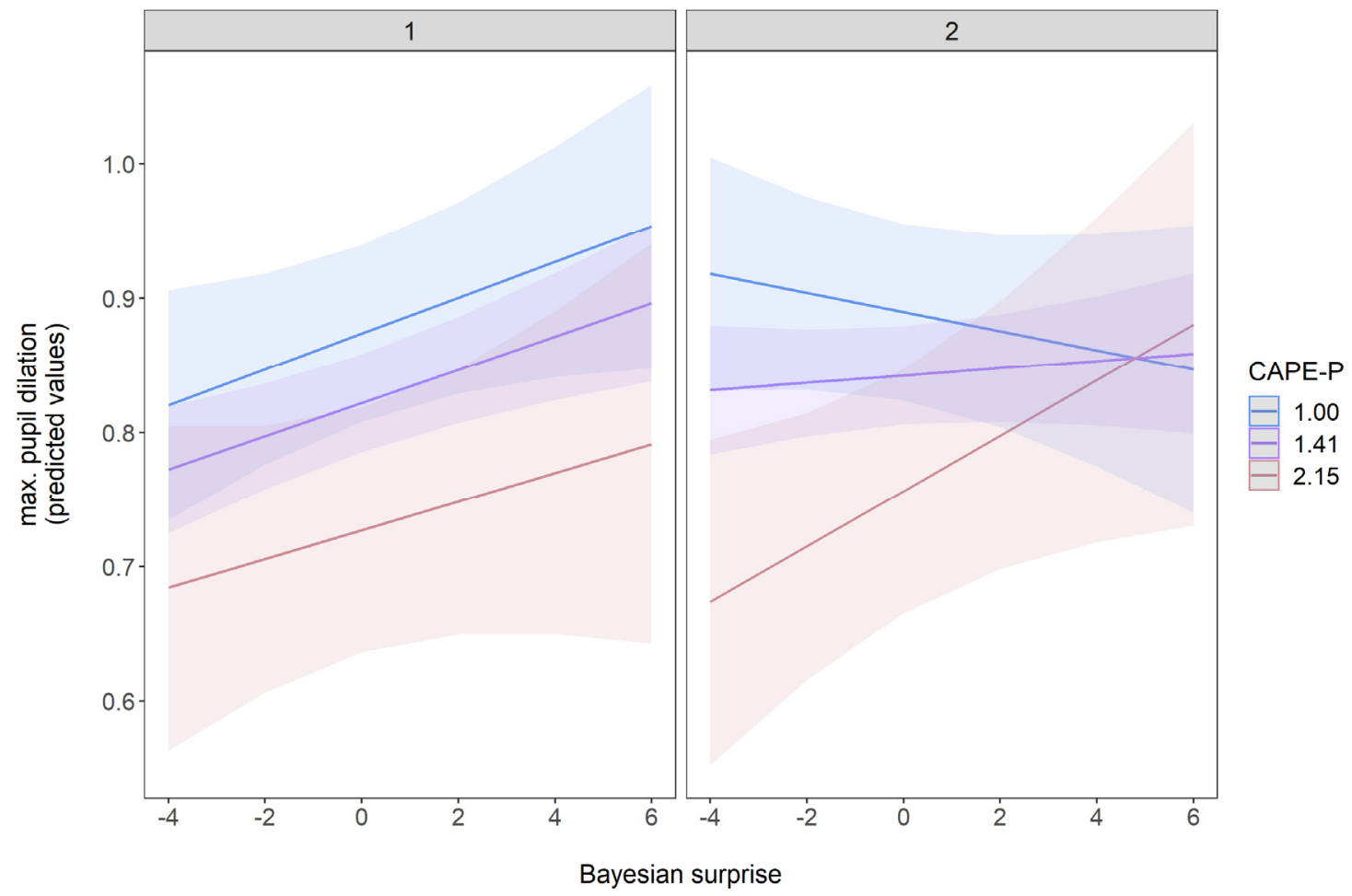




\section{References (Supplementary Information):}

Ahn, W. Y., Haines, N., \& Zhang, L. (2017). Revealing Neurocomputational Mechanisms of Reinforcement Learning and Decision-Making With the hBayesDM Package. Comput Psychiatr, 1, 24-57. https://doi.org/10.1162/CPSY_a_00002

den Ouden, Hanneke E. M., Daw, Nathaniel D., Fernandez, G., Elshout, Joris A., Rijpkema, M., Hoogman, M., . . . Cools, R. (2013). Dissociable Effects of Dopamine and Serotonin on Reversal Learning. Neuron, 80(4), 1090-1100. https://doi.org/https://doi.org/10.1016/j.neuron.2013.08.030

Gelman, A., Carlin, J. B., Stern, H. S., Dunson, D. B., Vehtari, A., \& Rubin, D. B. (2013). Bayesian data analysis. CRC press.

Gläscher, J., Hampton, A. N., \& O'Doherty, J. P. (2008). Determining a Role for Ventromedial Prefrontal Cortex in Encoding Action-Based Value Signals During Reward-Related Decision Making. Cerebral Cortex, 19(2), 483-495. https://doi.org/10.1093/cercor/bhn098

Kreis, I., Zhang, L., Moritz, S., \& Pfuhl, G. (2021). Spared performance but increased uncertainty in schizophrenia: Evidence from a probabilistic decision-making task. Schizophrenia https://doi.org/https://doi.org/10.1016/j.schres.2021.06.038

Lüdecke, D. (2021). sjPlot: Data Visualization for Statistics in Social Science, R package version 2.8.9. https://CRAN.R-project.org/package $=$ sjPlot

Nakagawa, S., \& Schielzeth, H. (2013). A general and simple method for obtaining R2 from generalized linear mixed-effects models. Methods in Ecology and Evolution, 4(2), 133-142. https://doi.org/10.1111/j.2041-210x.2012.00261.x

Pearce, J. M., \& Hall, G. (1980). A model for Pavlovian learning: Variations in the effectiveness of conditioned but not of unconditioned stimuli. Psychological Review, 87(6), 532-552. https://doi.org/10.1037/0033-295X.87.6.532

Rescorla, R. A., \& Wagner, A. R. (1972). A theory of Pavlovian conditioning: Variations in the effectiveness of reinforcement and nonreinforcement. In A. H. Black \& W. F. Prokasy (Eds.), Classical conditioning II: Current research and theory (pp. 6499). Appleton Century Crofts.

Schlagenhauf, F., Huys, Q. J. M., Deserno, L., Rapp, M. A., Beck, A., Heinze, H.-J., . . . Heinz, A. (2014). Striatal dysfunction during reversal learning in unmedicated $\begin{array}{llll}\text { schizophrenia } \quad \text { patients. } & \text { NeuroImage, }\end{array}$ https://doi.org/https://doi.org/10.1016/j.neuroimage.2013.11.034

Stan Development Team. (2016). RStan: the R interface to Stan. R package version, 2.16.2. https://doi.org/http://mc-stan.org/

Stone, J. M., \& Towse, J. N. (2015). A working memory test battery: Java-based collection of seven working memory tasks. Journal of Open Research Software, 3(1), e5. https://doi.org/http://doi.org/10.5334/jors.br

Worthy, D. A., \& Todd Maddox, W. (2014). A comparison model of reinforcementlearning and win-stay-lose-shift decision-making processes: A tribute to W.K. Estes. Journal of Mathematical Psychology, 59, 41-49. https://doi.org/https://doi.org/10.1016/j.jmp.2013.10.001 